\title{
Remark on the Lifespan of Solutions to 3-D Anisotropic Navier Stokes Equations
}

\author{
Siyu Liang ${ }^{1,2,4}$, Ping Zhang ${ }^{1,2, *}$ and Rongchan $\mathrm{Zhu}^{3,4}$ \\ ${ }^{1}$ Academy of Mathematics and Systems Science, Chinese Academy of \\ Sciences, Beijing 100190, China. \\ 2 School of Mathematical Sciences, University of Chinese Academy of \\ Sciences, Beijing 100049, China. \\ ${ }^{3}$ Department of Mathematics, Beijing Institute of Technology, Beijing 100081, \\ China. \\ ${ }^{4}$ Department of Mathematics, University of Bielefeld, D-33615 Bielefeld, \\ Germany.
}

Received 10 February 2020; Accepted 29 February 2020

\begin{abstract}
The goal of this article is to provide a lower bound for the lifespan of smooth solutions to 3-D anisotropic incompressible Navier-Stokes system, which in particular extends a similar type of result for the classical 3-D incompressible Navier-Stokes system.
\end{abstract}

AMS subject classifications: 35Q30, 76D03

Key words: Anisotropic Navier-Stokes system, Littlewood-Paley theory, lifespan.

\section{Introduction}

In this article, we shall investigate the lifespan for smooth enough solutions to the following 3-D anisotropic incompressible Navier-Stokes system:

$$
\left\{\begin{array}{l}
\partial_{t} u+u \cdot \nabla u-\Delta_{\mathrm{h}} u=-\nabla p, \\
\operatorname{div} u=0, \\
\left.u\right|_{t=0}=u_{0},
\end{array}\right.
$$

\footnotetext{
${ }^{*}$ Corresponding author. Email addresses: liangsiyu@amss.ac.cn (S. Liang), zp@math.ac.cn (P. Zhang), zhurongchan@126.com (R. Zhu)
} 
where $\Delta_{\mathrm{h}} \stackrel{\text { déf }}{=} \partial_{1}^{2}+\partial_{2}^{2}, u$ designates the velocity of the fluid and $p$ the scalar pressure function which guarantees the divergence free condition of the velocity field.

Systems of this type appear in geophysical fluid dynamics (see for instance $[5,16])$. In fact, meteorologists often modelize turbulent diffusion by putting a viscosity of the form: $-\mu_{\mathrm{h}} \Delta_{\mathrm{h}}-\mu_{3} \partial_{3}^{2}$, where $\mu_{\mathrm{h}}$ and $\mu_{3}$ are empirical constants, and $\mu_{3}$ is usually much smaller than $\mu_{\mathrm{h}}$. We refer to the book of Pedlovsky [16], Chap. 4 for a complete discussion about this model.

We remark that for the classical Navier-Stokes system (NS), which corresponds to (1.1) with $\Delta_{\mathrm{h}}$ there being replaced by $\Delta=\partial_{1}^{2}+\partial_{2}^{2}+\partial_{3}^{2}$, Leray [12] proved the global existence of weak solutions to $(N S)$ in 1934 . Yet the uniqueness and regularity to such weak solutions are still open. In [6], Chemin and Gallagher showed that: let $u_{0}$ be a regular solenoidal vector field, then the classical NavierStokes system $(N S)$ has a unique regular solution on $[0, T]$. Let $T^{\star}\left(u_{0}\right)$ be the maximal time of existence of this regular solution. Then for any $\gamma \in(0,1 / 4)$, a positive constant $C_{\gamma}$ exists so that

$$
T^{\star}\left(u_{0}\right) \geq C_{\gamma}\left\|u_{0}\right\|_{\dot{H}^{\frac{1}{2}+2 \gamma}}^{-\frac{1}{\gamma}}
$$

In the special case when $\gamma=\frac{1}{4}$, this type of result goes back to the seminal work of Leray [12]. Lately the same type of result has been proved for 3-D inhomogeneous incompressible Navier-Stokes system in [17] by the second author.

Considering that the system (1.1) has only horizontal dissipation, it is reasonable to use anisotropic Sobolev space defined as follows:

Definition 1.1. For any $\left(s, s^{\prime}\right)$ in $\mathbb{R}^{2}$, the anisotropic Sobolev space $\dot{H}^{s, s^{\prime}}\left(\mathbb{R}^{3}\right)$ denotes the space of homogeneous tempered distribution a such that

$$
\|a\|_{\dot{H}^{s, s^{\prime}}}^{2} \stackrel{\text { def }}{=} \int_{\mathbb{R}^{3}}\left|\xi_{\mathrm{h}}\right|^{2 s}\left|\xi_{3}\right|^{2 s^{\prime}}|\widehat{a}(\xi)|^{2} d \xi<\infty \quad \text { with } \quad \xi_{\mathrm{h}}=\left(\xi_{1}, \xi_{2}\right) .
$$

Mathematically, Chemin et al. [4] first studied the system (1.1). In particular, Chemin et al. [4] and Iftimie [11] proved that (1.1) is locally well-posed with initial data in $L^{2} \cap \dot{H}^{0, \frac{1}{2}+\varepsilon}$ for some $\varepsilon>0$, and is globally well-posed if in addition

$$
\left\|u_{0}\right\|_{L^{2}}^{\varepsilon}\left\|u_{0}\right\|_{\dot{H}^{0, \frac{1}{2}+\varepsilon}}^{1-\varepsilon} \leq c
$$

for some sufficiently small constant $c$.

Paicu [14] improved the well-posedness result in $[4,11]$ to be the critical case, namely, with initial data in the critical anisotropic Besov space, which basically 
corresponds to $\varepsilon=0$ in $[4,11]$. Chemin and the second author [8] introduced an anisotropic Besov-Sobolev type space with negative index and proved the global well-posedness of (1.1) with initial data being sufficiently small in this space. Paicu and the second author [15] improved further the global well-posedness result in [8] by requiring only two components of the initial data to be small in such negative anisotropic Besov spaces. Lately Liu and the second author proved the global well-posedness of (1.1) by requiring only one directional derivative of the initial data to be sufficiently small in some critical spaces. One may check [13] and the references therein concerning the recent progresses on the well-posedness of this system (1.1).

The goal of this article is to extend similar result as (1.2) for the lifespan of solutions to the classical Navier-Stokes system to the case of (1.1). The main result states as follows:

Theorem 1.1. Let $s=2 \gamma+\frac{1}{2}$ with $\gamma \in(0,1 / 4)$. Let $u_{0} \in H^{s}$ be a solenoidal vector field with $\partial_{3} u_{0} \in \dot{H}^{s-1,0} \cap \dot{H}^{-1, s}$. Then (1.1) has a unique solution $u \in C\left([0, T] ; H^{0, s}\right)$ with $\nabla_{\mathrm{h}} u \in L^{2}\left((0, T) ; \dot{H}^{0, s}\right)$ for some $T>0$. Moreover, if $T^{*}\left(u_{0}\right)$ designates the lifespan of this solution, there exists a positive constant $C_{\gamma}$ so that

$$
T^{*}\left(u_{0}\right) \geq C_{\gamma}\left[u_{0}\right]_{s}^{-\frac{1}{\gamma}} \quad \text { with } \quad\left[u_{0}\right]_{s}^{2} \stackrel{\text { def }}{=}\left\|u_{0}\right\|_{\dot{H}^{0, s}}^{2}+\left\|\partial_{3} u_{0}\right\|_{\dot{H}^{s-1,0}}^{2}+\left\|\partial_{3} u_{0}\right\|_{\dot{H}^{-1, s}}^{2} \text {. }
$$

Let us end this section with the notations that we shall use in this context.

Notations: Let $A, B$ be two operators, we denote $[A ; B]=A B-B A$, the commutator between $A$ and $B$, for $a \lesssim b$, we means that there is a uniform constant $C$, which may be different in each occurrence, such that $a \leq C b$. We shall denote by $(a \mid b)_{L^{2}}$ the $L^{2}\left(\mathbb{R}^{3}\right)$ inner product of $a$ and $b$. We always denote $\left(c_{\ell}\right)_{\ell \in \mathbb{Z}}$ to be a nonnegative generic element of $\ell^{2}(\mathbb{Z})$ so that $\sum c_{\ell}^{2}=1$. Finally, we denote $L_{T}^{r}\left(L_{\mathrm{h}}^{p}\left(L_{\mathrm{v}}^{q}\right)\right)$ the space $L^{r}\left([0, T] ; L^{p}\left(\mathbb{R}_{x_{1}} \times \mathbb{R}_{x_{2}} ; L^{q}\left(\mathbb{R}_{x_{3}}\right)\right)\right)$, and $\nabla_{\mathrm{h}} \stackrel{\text { def }}{=}\left(\partial_{x_{1}}, \partial_{x_{2}}\right), \operatorname{div}_{\mathrm{h}}=\partial_{x_{1}}+\partial_{x_{2}}$.

\section{Littlewood-Paley analysis and product laws}

In the rest of this paper, we shall frequently use Littlewood-Paley decomposition in the vertical variable. For the convenience of the readers, we collect some basic facts on anisotropic Littlewood-Paley theory in this section. Let us first recall from [1] that

$$
\begin{array}{rlrl}
\Delta_{k}^{\mathrm{h}} a & =\mathcal{F}^{-1}\left(\varphi\left(2^{-k}\left|\xi_{\mathrm{h}}\right|\right) \widehat{a}\right), & & S_{k}^{\mathrm{h}} a=\mathcal{F}^{-1}\left(\chi\left(2^{-k}\left|\xi_{\mathrm{h}}\right|\right) \widehat{a}\right), \\
\Delta_{\ell}^{\mathrm{v}} a=\mathcal{F}^{-1}\left(\varphi\left(2^{-\ell}\left|\xi_{3}\right|\right) \widehat{a}\right), & S_{\ell}^{\mathrm{v}} a=\mathcal{F}^{-1}\left(\chi\left(2^{-\ell}\left|\xi_{3}\right|\right) \widehat{a}\right),
\end{array}
$$


where $\xi=\left(\xi_{h}, \xi_{3}\right), \mathcal{F} a$ and $\widehat{a}$ denote the Fourier transform of the distribution $a$, and $\chi(\tau), \varphi(\tau)$ are smooth functions such that

$$
\begin{array}{lll}
\text { Supp } \varphi \subset\left\{\tau \in \mathbb{R} / \frac{3}{4} \leq|\tau| \leq \frac{8}{3}\right\} & \text { and } & \forall \tau>0, \sum_{k \in \mathbb{Z}} \varphi\left(2^{-k} \tau\right)=1, \\
\text { Supp } \chi \subset\left\{\tau \in \mathbb{R} /|\tau| \leq \frac{4}{3}\right\} & \text { and } & \chi(\tau)+\sum_{k \geq 0} \varphi\left(2^{-k} \tau\right)=1 .
\end{array}
$$

Next we recall the following anisotropic Bernstein inequalities from $[8,14]$ :

Lemma 2.1. Let $\mathbf{B}_{\mathrm{h}}$ (resp. $\mathbf{B}_{\mathrm{v}}$ ) a ball of $\mathbb{R}_{\mathrm{h}}^{2}$ (resp. $\mathbb{R}_{\mathrm{v}}$ ), and $\mathcal{C}_{\mathrm{h}}$ (resp. $\mathcal{C}_{\mathrm{v}}$ ) a ring of $\mathbb{R}_{\mathrm{h}}^{2}$ (resp. $\mathbb{R}_{\mathrm{v}}$ ); let $1 \leq p_{2} \leq p_{1} \leq \infty$ and $1 \leq q_{2} \leq q_{1} \leq \infty$. Then there holds

$$
\begin{aligned}
& \text { if Supp } \widehat{a} \subset 2^{k} \mathbf{B}_{\mathrm{h}} \Rightarrow\left\|\partial_{x_{\mathrm{h}}}^{\alpha} a\right\|_{L_{\mathrm{h}}^{p_{1}}\left(L_{\mathrm{v}}^{q_{1}}\right)} \lesssim 2^{k\left(|\alpha|+\frac{2}{p_{2}}-\frac{2}{p_{1}}\right)}\|a\|_{L_{\mathrm{h}}^{p_{2}}\left(L_{\mathrm{v}}^{q_{1}}\right)^{\prime}} \\
& \text { if Supp } \widehat{a} \subset 2^{\ell} \mathbf{B}_{\mathrm{v}} \Rightarrow\left\|\partial_{x_{3}}^{\beta} a\right\|_{L_{\mathrm{h}}^{p_{1}}\left(L_{\mathrm{v}}^{q_{1}}\right)} \lesssim 2^{\ell\left(\beta+\frac{1}{q_{2}}-\frac{1}{q_{1}}\right)}\|a\|_{L_{\mathrm{h}}^{p_{1}}\left(L_{\mathrm{v}}^{q_{2}}\right)^{\prime}} ; \\
& \text { if Supp } \widehat{a} \subset 2^{k} \mathcal{C}_{\mathrm{h}} \Rightarrow\|a\|_{L_{\mathrm{h}}^{p_{1}}\left(L_{\mathrm{v}}^{q_{1}}\right)} \lesssim 2^{-k N} \sup _{|\alpha|=N}\left\|\partial_{x_{\mathrm{h}}}^{\alpha} a\right\|_{L_{\mathrm{h}}^{p_{1}}\left(L_{\mathrm{v}}^{q_{1}}\right)^{\prime}} \\
& \text { if Supp } \widehat{a} \subset 2^{\ell} \mathcal{C}_{\mathrm{v}} \Rightarrow\|a\|_{L_{\mathrm{h}}^{p_{1}}\left(L_{\mathrm{v}}^{q_{1}}\right)} \lesssim 2^{-\ell N}\left\|\partial_{x_{3}}^{N} a\right\|_{L_{\mathrm{h}}^{p_{1}}\left(L_{\mathrm{v}}^{q_{1}}\right)^{\prime}}
\end{aligned}
$$

Before preceding, we recall Bony's decomposition for the vertical variable from [2]:

$$
a b=T_{a}^{\mathrm{v}} b+R^{\mathrm{v}}(a, b) \quad \text { with } \quad T_{a}^{\mathrm{v}} b=\sum_{\ell \in \mathbb{Z}} S_{\ell-1}^{\mathrm{v}} a \Delta_{\ell}^{\mathrm{v}} b, \quad R^{\mathrm{v}}(a, b)=\sum_{\ell \in \mathbb{Z}} \Delta_{\ell}^{\mathrm{V}} a S_{\ell+2}^{\mathrm{v}} b .
$$

Let us now apply the above basic facts on Littlewood-Paley theory to prove the following laws of product:

Lemma 2.2. Let $s \in\left(\frac{1}{2}, 1\right)$, one has

$$
\begin{aligned}
\left\|\Delta_{\ell}^{\mathrm{v}}(a b)\right\|_{L_{\mathrm{v}}^{2}\left(L_{\mathrm{h}}^{\frac{4}{3}}\right)} \lesssim & c_{\ell} 2^{-\ell s}\left(\|a\|_{\dot{H}^{0, s}}^{\frac{1}{2}}\left\|\nabla_{\mathrm{h}} a\right\|_{\dot{H}^{0, s}}^{\frac{1}{2}}\|b\|_{L^{2}}^{1-\frac{1}{2 s}}\|b\|_{\dot{H}^{0, s}}^{\frac{1}{2 s}}\right. \\
& \left.+\left(\|a\|_{L^{2}}\left\|\nabla_{\mathrm{h}} a\right\|_{L^{2}}\right)^{\frac{1}{2}-\frac{1}{4 s}}\left(\|a\|_{\dot{H}^{0, s}}\left\|\nabla_{\mathrm{h}} a\right\|_{\dot{H}^{0, s}}\right)^{\frac{1}{4 s}}\|b\|_{\dot{H}^{0, s}}\right) .
\end{aligned}
$$

Proof. By applying Bony's decomposition (2.2) in the vertical variable to $a b$, we find

$$
a b=T_{a}^{\mathrm{v}} b+R^{\mathrm{v}}(a, b) .
$$


Considering the support properties to the Fourier transform of the terms in $T_{a}^{\mathrm{v}} b$, we write

$$
\begin{aligned}
\left\|\Delta_{\ell}^{\mathrm{v}}\left(T_{a}^{\mathrm{v}} b\right)\right\|_{L_{\mathrm{V}}^{2}\left(L_{\mathrm{h}}^{\frac{4}{3}}\right)} & \lesssim \sum_{|j-\ell| \leq 5}\left\|S_{j-1}^{\mathrm{v}} a\right\|_{L_{\mathrm{v}}^{\infty}\left(L_{\mathrm{h}}^{4}\right)}\left\|\Delta_{j}^{\mathrm{v}} b\right\|_{L^{2}} \\
& \lesssim\|a\|_{L_{\mathrm{v}}^{\infty}\left(L_{\mathrm{h}}^{4}\right)}\left(\sum_{|j-\ell| \leq 5}\left\|\Delta_{j}^{\mathrm{v}} b\right\|_{L^{2}}\right) \\
& \lesssim\|a\|_{L_{\mathrm{v}}^{\infty}\left(L_{\mathrm{h}}^{2}\right)}^{\frac{1}{2}}\left\|\nabla_{\mathrm{h}} a\right\|_{L_{\mathrm{v}}^{\infty}\left(L_{\mathrm{h}}^{2}\right)}^{\frac{1}{2}}\left(\sum_{|j-\ell| \leq 5}\left\|\Delta_{j}^{\mathrm{v}} b\right\|_{L^{2}}\right),
\end{aligned}
$$

from which, and

$$
\|f\|_{L_{\mathrm{v}}^{\infty}\left(L_{\mathrm{h}}^{2}\right)} \lesssim\|f\|_{L_{\mathrm{h}}^{2}\left(L_{\mathrm{v}}^{\infty}\right)} \lesssim\|f\|_{L_{\mathrm{h}}^{2}\left(B_{2,1}^{\frac{1}{2}}\right)_{\mathrm{v}}} \lesssim\|f\|_{L^{2}}^{1-\frac{1}{2 s}}\|f\|_{H^{0, s}}^{\frac{1}{2 s}}
$$

We deduce that

$$
\left\|\Delta_{\ell}^{\mathrm{v}}\left(T_{a}^{\mathrm{v}} b\right)\right\|_{L_{\mathrm{v}}^{2}\left(L_{\mathrm{h}}^{\frac{4}{3}}\right)} \lesssim c_{\ell} 2^{-\ell s}\left(\|a\|_{L^{2}}\left\|\nabla_{\mathrm{h}} a\right\|_{L^{2}}\right)^{\frac{1}{2}-\frac{1}{4 s}}\left(\|a\|_{\dot{H}^{0, s}}\left\|\nabla_{\mathrm{h}} a\right\|_{\dot{H}^{0, s}}\right)^{\frac{1}{4 s}}\|b\|_{\dot{H}^{0, s}}
$$

Along the same line to the proof of (2.5), we infer

$$
\begin{aligned}
\left\|\Delta_{\ell}^{\mathrm{v}}\left(R^{\mathrm{v}}(a, b)\right)\right\|_{L_{\mathrm{v}}^{2}\left(L_{\mathrm{h}}^{\frac{4}{3}}\right)} & \lesssim \sum_{j \geq \ell-N_{0}}\left\|S_{j+2}^{\mathrm{v}} b\right\|_{L_{\mathrm{v}}^{\infty}\left(L_{\mathrm{h}}^{2}\right)}\left\|\Delta_{j}^{\mathrm{V}} a\right\|_{L_{\mathrm{V}}^{2}\left(L_{\mathrm{h}}^{4}\right)} \\
& \lesssim\|b\|_{L_{\mathrm{v}}^{\infty}\left(L_{\mathrm{h}}^{2}\right)}\left(\sum_{j \geq \ell-N_{0}}\left\|\Delta \Delta_{j}^{\mathrm{v}} a\right\|_{L_{\mathrm{V}}^{2}\left(L_{\mathrm{h}}^{4}\right)}\right) \\
& \lesssim\|b\|_{L^{2}}^{1-\frac{1}{2 s}}\|b\|_{\dot{H}^{0, s}}^{\frac{1}{2 s}}\left(\sum_{j \geq \ell-N_{0}}\left\|\Delta_{j}^{\mathrm{V}} a\right\|_{L^{2}}^{\frac{1}{2}}\left\|\Delta_{j}^{\mathrm{v}} \nabla_{\mathrm{h}} a\right\|_{L^{2}}^{\frac{1}{2}}\right) \\
& \lesssim\|b\|_{L^{2}}^{1-\frac{1}{2 s}}\|b\|_{\dot{H}^{0, s}}^{\frac{1}{2 s}}\|a\|_{\dot{H}^{0, s}}^{\frac{1}{2}}\left\|\nabla_{\mathrm{h}} a\right\|_{\dot{H}^{0, s}}^{\frac{1}{2}} \sum_{j \geq \ell-N_{0}} c_{j} 2^{-j s} .
\end{aligned}
$$

Notice that

$$
\left\|\left(2^{\ell s} \sum_{j \geq \ell-5} c_{j} 2^{-j s}\right)_{\ell \in \mathbb{Z}}\right\|_{\ell^{2}} \lesssim\left\|1_{(-\infty, 5]^{2 \cdot}}\right\|_{\ell^{1}}\left\|c_{j}\right\|_{\ell^{2}} \lesssim 1,
$$

we conclude that

$$
\left\|\Delta_{\ell}^{\mathrm{v}}\left(R^{\mathrm{v}}(a, b)\right)\right\|_{L_{\mathrm{v}}^{2}\left(L_{\mathrm{h}}^{\frac{4}{3}}\right)} \lesssim c_{\ell} 2^{-\ell s}\|b\|_{L^{2}}^{1-\frac{1}{2 s}}\|b\|_{H^{0, s}}^{\frac{1}{2 s}}\|a\|_{H^{0, s}}^{\frac{1}{2}}\left\|\nabla_{\mathrm{h}} a\right\|_{H^{0, s}}^{\frac{1}{2}} .
$$

Combining (2.5) with (2.7) leads to (2.3). 
Remark 2.1. The proof of Lemma 2.2 also implies that

$$
\left\|\Delta_{\ell}^{\mathrm{v}}(a b)\right\|_{L_{\mathrm{V}}^{2}\left(L_{\mathrm{h}}^{3}\right)}^{\frac{4}{3}} \lesssim c_{\ell} 2^{-\ell s}\left(\|a\|_{L_{\mathrm{V}}^{\infty}\left(L_{h}^{4}\right)}\|b\|_{\dot{H}^{0, s}}+\|a\|_{\dot{H}^{0, s}}^{\frac{1}{2}}\left\|\nabla_{\mathrm{h}} a\right\|_{\dot{H}^{0, s}}^{\frac{1}{2}}\|b\|_{L^{2}}^{1-\frac{1}{2 s}}\|b\|_{\dot{H}^{0, s}}^{\frac{1}{2 s}}\right),
$$

and

$$
\left\|\Delta_{\ell}^{\mathrm{v}}(a b)\right\|_{L_{\mathrm{v}}^{2}\left(L_{\mathrm{h}}^{\frac{4}{3}}\right)} \lesssim c_{\ell} 2^{-\ell s}\left(\|a\|_{L_{\mathrm{v}}^{\infty}\left(L_{h}^{4}\right)}\|b\|_{\dot{H}^{0, s}}+\|a\|_{\dot{H}^{0, s}}^{\frac{1}{2}}\left\|\nabla_{\mathrm{h}} a\right\|_{\dot{H}^{0, s}}^{\frac{1}{2}}\|b\|_{L_{\mathrm{v}}^{\infty}\left(L_{\mathrm{h}}^{2}\right)}\right) .
$$

Lemma 2.3. Let $a=\left(a^{\mathrm{h}}, a^{3}\right)$ be a solenoidal vector field. Then for any $s \in\left(\frac{1}{2}, 1\right)$, one has

$$
\begin{aligned}
& \left|\left(\Delta_{\ell}^{\mathrm{v}}\left(a^{3} \partial_{3} b\right) \mid \Delta_{\ell}^{\mathrm{v}} b\right)_{L^{2}}\right| \\
\lesssim & c_{\ell}^{2} 2^{-2 \ell s}\left(\left\|\nabla_{\mathrm{h}} a^{\mathrm{h}}\right\|_{L^{2}}^{1-\frac{1}{2 s}}\left\|\nabla_{\mathrm{h}} a^{\mathrm{h}}\right\|_{\dot{H}^{0, s}}^{\frac{1}{2 s}}\|b\|_{\dot{H}^{0, s}}\left\|\nabla_{\mathrm{h}} b\right\|_{\dot{H}^{0, s}}\right. \\
& \left.+\left\|\nabla_{\mathrm{h}} a^{\mathrm{h}}\right\|_{\dot{H}^{0, s}}\left(\|b\|_{L^{2}}\left\|\nabla_{\mathrm{h}} b\right\|_{L^{2}}\right)^{\frac{1}{2}-\frac{1}{4 s}}\left(\|b\|_{\dot{H}^{0, s}}\left\|\nabla_{\mathrm{h}} b\right\|_{\dot{H}^{0, s}}\right)^{\frac{1}{2}+\frac{1}{4 s}}\right) .
\end{aligned}
$$

Proof. By applying Bony's decomposition (2.2) in the vertical variable to $a^{3} \partial_{3} b$, we write

$$
a^{3} \partial_{3} b=T_{a^{3}}^{\mathrm{v}} \partial_{3} b+R^{\mathrm{v}}\left(a^{3}, \partial_{3} b\right) .
$$

We first deduce from the support properties to the Fourier transform of the terms in $R^{\mathrm{v}}\left(a^{3}, \partial_{3} b\right)$ and Lemma 2.1 that

$$
\begin{aligned}
& \left|\left(\Delta_{\ell}^{\mathrm{v}}\left(R^{\mathrm{v}}\left(a^{3}, \partial_{3} b\right)\right) \mid \Delta_{\ell}^{\mathrm{v}} b\right)_{L^{2}}\right| \\
\lesssim & \sum_{j \geq \ell-N_{0}} 2^{j}\left\|\Delta_{j}^{\mathrm{v}} a^{3}\right\|_{L^{2}}\left\|S_{j+2}^{v} b\right\|_{L_{\mathrm{v}}^{\infty}\left(L_{\mathrm{h}}^{4}\right)}\left\|\Delta_{\ell}^{\mathrm{v}} b\right\|_{L_{\mathrm{v}}^{2}\left(L_{\mathrm{h}}^{4}\right)} \\
\lesssim & \sum_{j \geq \ell-N_{0}}\left\|\Delta_{j}^{\mathrm{v}} \partial_{3} a^{3}\right\|_{L^{2}}\|b\|_{L_{\mathrm{v}}^{\infty}\left(L_{\mathrm{h}}^{2}\right)}^{\frac{1}{2}}\left\|\nabla_{\mathrm{h}} b\right\|_{L_{\mathrm{v}}^{\infty}\left(L_{\mathrm{h}}^{2}\right)}^{\frac{1}{2}}\left\|\Delta_{\ell}^{\mathrm{v}} b\right\|_{L^{2}}^{\frac{1}{2}}\left\|\Delta_{\ell}^{\mathrm{v}} \nabla_{\mathrm{h}} b\right\|_{L^{2}}^{\frac{1}{2}},
\end{aligned}
$$

which together with (2.4) and $\partial_{3} a^{3}=-\operatorname{div}_{\mathrm{h}} a^{\mathrm{h}}$ ensures that

$$
\begin{aligned}
& \left|\left(\Delta_{\ell}^{\mathrm{v}}\left(R^{\mathrm{v}}\left(a^{3}, \partial_{3} b\right)\right) \mid \Delta_{\ell}^{\mathrm{v}} b\right)_{L^{2}}\right| \\
\lesssim & c_{\ell} 2^{-\ell s}\left(\|b\|_{L^{2}}\left\|\nabla_{\mathrm{h}} b\right\|_{L^{2}}\right)^{\frac{1}{2}-\frac{1}{4 s}}\left(\|b\|_{\dot{H}^{0, s}}\left\|\nabla_{\mathrm{h}} b\right\|_{\dot{H}^{0, s}}\right)^{\frac{1}{2}+\frac{1}{4 s}}\left\|\nabla_{\mathrm{h}} a^{\mathrm{h}}\right\|_{\dot{H}^{0, s}} \sum_{j \geq \ell-N_{0}} c_{j} 2^{-j s} \\
\lesssim & c_{\ell}^{2} 2^{-2 \ell s}\left(\|b\|_{L^{2}}\left\|\nabla_{\mathrm{h}} b\right\|_{L^{2}}\right)^{\frac{1}{2}-\frac{1}{4 s}}\left(\|b\|_{\dot{H}^{0, s}}\left\|\nabla_{\mathrm{h}} b\right\|_{\dot{H}^{0, s}}\right)^{\frac{1}{4 s}+\frac{1}{2}}\left\|\nabla_{\mathrm{h}} a^{\mathrm{h}}\right\|_{\dot{H}^{0, s}}
\end{aligned}
$$

where in the last step we used (2.6) once again. 
To handle the term $\left(\Delta_{\ell}^{\mathrm{v}}\left(T_{a^{3}}^{\mathrm{v}} \partial_{3} b\right) \mid \Delta_{\ell}^{\mathrm{v}} b\right)_{L^{2}}$, we get, by using a standard commutator's process (see for instance [4]), that

$$
\begin{aligned}
\Delta_{\ell}^{\mathrm{v}}\left(T_{a^{3}} \partial_{3} b\right)= & S_{\ell-1}^{\mathrm{V}} a^{3} \partial_{3} \Delta_{\ell}^{\mathrm{v}} b+\sum_{|j-\ell| \leq 5}\left[\Delta_{\ell}^{\mathrm{V}} ; S_{j-1}^{\mathrm{V}} a^{3}\right] \partial_{3} \Delta_{j}^{\mathrm{V}} b \\
& +\sum_{|j-\ell| \leq 5}\left(S_{j-1}^{\mathrm{v}} a^{3}-S_{\ell-1}^{\mathrm{v}} a^{3}\right) \partial_{3} \Delta_{\ell}^{\mathrm{v}} \Delta_{j}^{\mathrm{V}} b .
\end{aligned}
$$

Corresponding to the first term in (2.12), we get, by applying (2.4) and $\operatorname{div} a=0$, that

$$
\begin{aligned}
\left|\left(S_{\ell-1}^{\mathrm{v}} a^{3} \partial_{3} \Delta_{\ell}^{\mathrm{v}} b \mid \Delta_{\ell}^{\mathrm{v}} b\right)_{L^{2}}\right| & =\frac{1}{2}\left|\left(S_{\ell-1}^{\mathrm{v}} \partial_{3} a^{3} \Delta_{\ell}^{\mathrm{v}} b \mid \Delta_{\ell}^{\mathrm{v}} b\right)_{L^{2}}\right| \\
& \lesssim\left\|\partial_{3} a^{3}\right\|_{L_{\mathrm{v}}^{\infty}\left(L_{\mathrm{h}}^{2}\right)}\left\|\Delta_{\ell}^{\mathrm{v}} b\right\|_{L_{\mathrm{v}}^{2}\left(L_{\mathrm{h}}^{4}\right)}^{2} \\
& \lesssim\left\|\nabla_{\mathrm{h}} a^{\mathrm{h}}\right\|_{L_{\mathrm{v}}^{\infty}\left(L_{\mathrm{h}}^{2}\right)}\left\|\Delta_{\ell}^{\mathrm{v}} b\right\|_{L^{2}}\left\|\Delta_{\ell}^{\mathrm{v}} \nabla_{\mathrm{h}} b\right\|_{L^{2}} \\
& \lesssim c_{\ell}^{2} 2^{-2 \ell s}\left\|\nabla_{\mathrm{h}} a^{\mathrm{h}}\right\|_{L^{2}}^{1-\frac{1}{2 s}}\left\|\nabla_{\mathrm{h}} a^{\mathrm{h}}\right\|_{\dot{H}^{0, s}}^{\frac{1}{2 s}}\|b\|_{\dot{H}^{0, s}}\left\|\nabla_{\mathrm{h}} b\right\|_{\dot{H}^{0, s}} .
\end{aligned}
$$

Similarly, corresponding to the last term in (2.12), we get, by applying Lemma 2.1 , that

$$
\begin{aligned}
& \left.\left|\sum_{|j-\ell| \leq 5}\left(S_{j-1}^{\mathrm{v}} a^{3}-S_{\ell-1}^{\mathrm{v}} a^{3}\right) \partial_{3} \Delta_{\ell}^{\mathrm{v}} \Delta_{j}^{\mathrm{v}} b\right| \Delta_{\ell}^{\mathrm{v}} b\right)_{L^{2}} \mid \\
\lesssim & \sum_{|j-\ell| \leq 5}\left\|S_{j-1}^{\mathrm{v}} a^{3}-S_{\ell-1}^{\mathrm{v}} a^{3}\right\|_{L_{\mathrm{v}}^{\infty}\left(L_{\mathrm{h}}^{2}\right)}\left\|\partial_{3} \Delta_{\ell}^{\mathrm{v}} b\right\|_{L_{\mathrm{v}}^{2}\left(L_{\mathrm{h}}^{4}\right)}\left\|\Delta_{\ell}^{\mathrm{v}} b\right\|_{L_{\mathrm{v}}^{2}\left(L_{\mathrm{h}}^{4}\right)} \\
\lesssim & \sum_{|j-\ell| \leq 5}\left\|\Delta_{j}^{\mathrm{v}} a^{3}\right\|_{L_{\mathrm{v}}^{\infty}\left(L_{\mathrm{h}}^{2}\right.} 2^{\ell}\left\|\Delta_{\ell}^{\mathrm{v}} b\right\|_{L_{\mathrm{v}}^{2}\left(L_{\mathrm{h}}^{4}\right)}^{2} \\
\lesssim & \sum_{|j-\ell| \leq 5}\left\|\Delta_{j}^{\mathrm{v}} \partial_{3} a^{3}\right\|_{L_{\mathrm{v}}^{\infty}\left(L_{\mathrm{h}}^{2}\right)}\left\|\Delta_{\ell}^{\mathrm{v}} b\right\|_{L^{2}}\left\|\Delta_{\ell}^{\mathrm{v}} \nabla_{\mathrm{h}} b\right\|_{L^{2}} \\
\lesssim & c_{\ell}^{2} 2^{-2 \ell s}\left\|\nabla_{\mathrm{h}} a^{\mathrm{h}}\right\|_{L^{2}}^{1-\frac{1}{2 s}}\left\|\nabla_{\mathrm{h}} a^{\mathrm{h}}\right\|_{H^{0, s}}^{\frac{1}{2 s}}\|b\|_{\dot{H}^{0, s}}\left\|\nabla_{\mathrm{h}} b\right\|_{\dot{H}^{0, s}} .
\end{aligned}
$$

Finally let us deal with the commutator term. We observe that

$$
\begin{aligned}
& \sum_{|j-\ell| \leq 5}\left|\left(\left[\Delta_{\ell}^{\mathrm{v}} ; S_{j-1}^{\mathrm{v}} a^{3}\right] \partial_{3} \Delta_{j}^{\mathrm{v}} b \mid \Delta_{\ell}^{\mathrm{v}} b\right)_{L^{2}}\right| \\
\lesssim & \sum_{|j-\ell| \leq 5}\left\|\left[\Delta_{\ell}^{\mathrm{v}} ; S_{j-1}^{\mathrm{v}} a^{3}\right] \partial_{3} \Delta_{j}^{\mathrm{v}} b\right\|_{L_{\mathrm{v}}^{2}\left(L_{\mathrm{h}}^{\frac{4}{3}}\right)}\left\|\Delta_{\ell}^{\mathrm{v}} b\right\|_{L_{\mathrm{v}}^{2}\left(L_{\mathrm{h}}^{4}\right)} .
\end{aligned}
$$


Let $h \stackrel{\text { def }}{=} \mathcal{F}^{-1} \varphi$ and $h_{1}(z) \stackrel{\text { def }}{=} z h(z)$. Then again due to $\partial_{3} a^{3}=-\operatorname{div}_{\mathrm{h}} a^{\mathrm{h}}$, we find

$$
\begin{aligned}
& \left|\left[\Delta_{\ell}^{\mathrm{v}} ; S_{j-1}^{\mathrm{v}} a^{3}\right] \partial_{3} \Delta_{j}^{\mathrm{v}} b\left(x_{\mathrm{h}}, x_{3}\right)\right| \\
= & 2^{\ell}\left|\int_{\mathbb{R}} h\left(2^{\ell} y_{3}\right)\left(S_{j-1}^{\mathrm{v}} a^{3}\left(x_{\mathrm{h}}, x_{3}-y_{3}\right)-S_{j-1}^{\mathrm{v}} a^{3}\left(x_{\mathrm{h}}, x_{3}\right)\right) \partial_{3} \Delta_{j}^{\mathrm{v}} b\left(x_{\mathrm{h}}, x_{3}-y_{3}\right) d y_{3}\right| \\
= & 2^{\ell}\left|\int_{\mathbb{R} \times[0,1]} y_{3} h\left(2^{\ell} y_{3}\right) S_{j-1}^{\mathrm{v}} \partial_{3} a^{3}\left(x_{\mathrm{h}}, x_{3}-\tau y_{3}\right) \partial_{3} \Delta_{j}^{\mathrm{v}} b\left(x_{\mathrm{h}}, x_{3}-y_{3}\right) d \tau d y_{3}\right| \\
= & \left|\int_{\mathbb{R} \times[0,1]} h_{1}\left(2^{\ell} y_{3}\right) S_{j-1}^{\mathrm{v}} \operatorname{div}_{\mathrm{h}} a^{h}\left(x_{\mathrm{h}}, x_{3}-\tau y_{3}\right) \partial_{3} \Delta_{j}^{\mathrm{v}} b\left(x_{\mathrm{h}}, x_{3}-y_{3}\right) d \tau d y_{3}\right| .
\end{aligned}
$$

When $|j-\ell| \leq 5$, taking the $L_{\mathrm{v}}^{2}\left(L_{\mathrm{h}}^{\frac{4}{3}}\right)$ norm to the above quantity gives rise to

$$
\begin{aligned}
& \left\|\left[\Delta_{\ell}^{\mathrm{v}} ; S_{j-1}^{\mathrm{v}} a^{3}\right] \partial_{3} \Delta_{j}^{\mathrm{v}} b\right\|_{L_{\mathrm{v}}^{2}\left(L_{\mathrm{h}}^{\frac{4}{3}}\right)} \\
\lesssim & \int_{\mathbb{R} \times[0,1]}\left|h_{1}\left(2^{\ell} y_{3}\right)\right|\left\|S_{j-1}^{\mathrm{v}} \operatorname{div}_{\mathrm{h}} a^{h}\left(\cdot, \cdot-\tau y_{3}\right)\right\|_{L_{\mathrm{v}}^{\infty}\left(L_{\mathrm{h}}^{2}\right)}\left\|\partial_{3} \Delta_{j}^{\mathrm{v}} b\left(\cdot, \cdot-y_{3}\right)\right\|_{L_{\mathrm{v}}^{2}\left(L_{\mathrm{h}}^{4}\right)} d \tau d y_{3} \\
= & 2^{\ell}\left\|S_{j-1}^{\mathrm{v}} \operatorname{div}_{\mathrm{h}} a^{h}(\cdot, \cdot)\right\|_{L_{\mathrm{v}}^{\infty}\left(L_{\mathrm{h}}^{2}\right)}\left\|\Delta_{j}^{\mathrm{v}} b\right\|_{L_{\mathrm{v}}^{2}\left(L_{\mathrm{h}}^{4}\right)} \int_{\mathbb{R}}\left|h_{1}\left(2^{\ell} y_{3}\right)\right| d y_{3} \\
\lesssim & \left\|\nabla_{\mathrm{h}} a^{\mathrm{h}}\right\|_{L_{\mathrm{V}}^{\infty}\left(L_{\mathrm{h}}^{2}\right)}\left\|\Delta_{j}^{\mathrm{v}} b\right\|_{L^{2}}^{\frac{1}{2}}\left\|\Delta_{j}^{\mathrm{v}} \nabla_{\mathrm{h}} b\right\|_{L^{2}}^{\frac{1}{2}} .
\end{aligned}
$$

Then by virtue of (2.4), we deduce that

$$
\begin{aligned}
& \sum_{|j-\ell| \leq 5}\left|\left(\left[\Delta_{\ell}^{\mathrm{v}} ; S_{j-1}^{\mathrm{v}} a^{3}\right] \partial_{3} \Delta_{j}^{\mathrm{v}} b \mid \Delta_{\ell}^{\mathrm{v}} b\right)_{L^{2}}\right| \\
& \lesssim c_{\ell}^{2} 2^{-2 \ell s}\left\|\nabla_{\mathrm{h}} a^{\mathrm{h}}\right\|_{L^{2}}^{1-\frac{1}{2 s}}\left\|\nabla_{\mathrm{h}} a^{\mathrm{h}}\right\|_{\dot{H}^{0, s}}^{\frac{1}{2 s}}\|b\|_{\dot{H}^{0, s}}\left\|\nabla_{\mathrm{h}} b\right\|_{\dot{H}^{0, s}} .
\end{aligned}
$$

As a result, it comes out

$$
\left|\left(\Delta_{\ell}^{\mathrm{v}}\left(T_{a^{3}}^{\mathrm{v}} \partial_{3} b\right) \mid \Delta_{\ell}^{\mathrm{v}} b\right)_{L^{2}}\right| \lesssim c_{\ell}^{2} 2^{-2 \ell s}\left\|\nabla_{\mathrm{h}} a^{\mathrm{h}}\right\|_{L^{2}}^{1-\frac{1}{2 s}}\left\|\nabla_{\mathrm{h}} a^{\mathrm{h}}\right\|_{\dot{H}^{0, s}}^{\frac{1}{2 s}}\|b\|_{\dot{H}^{0, s}}\left\|\nabla_{\mathrm{h}} b\right\|_{\dot{H}^{0, s}} .
$$

Along with (2.11), we complete the proof of (2.10).

Remark 2.2. The proof of Lemma 2.3 implies that

$$
\begin{aligned}
& \quad\left|\left(\Delta_{\ell}^{\mathrm{v}}\left(a^{3} \partial_{3} b\right) \mid \Delta_{\ell}^{\mathrm{v}} b\right)_{\dot{H}^{0, s}}\right| \\
& \lesssim c_{\ell}^{2} 2^{-2 \ell s}\left(\left\|\nabla_{\mathrm{h}} a^{\mathrm{h}}\right\|_{L_{\mathrm{v}}^{\infty}\left(L^{2}\right)}\|b\|_{\dot{H}^{0, s}}\left\|\nabla_{\mathrm{h}} b\right\|_{\dot{H}^{0, s}}\right. \\
& \left.\quad+\left\|\nabla_{\mathrm{h}} a^{\mathrm{h}}\right\|_{\dot{H}^{0, s}}\left(\|b\|_{L^{2}}\left\|\nabla_{\mathrm{h}} b\right\|_{L^{2}}\right)^{\frac{1}{2}-\frac{1}{4 s}}\left(\|b\|_{\dot{H}^{0, s}}\left\|\nabla_{\mathrm{h}} b\right\|_{\dot{H}^{0, s}}\right)^{\frac{1}{2}+\frac{1}{4 s}}\right) .
\end{aligned}
$$




\section{Scaled $L^{2}$ energy estimate}

Inspired by the lecture notes of Chemin [3], we are going to present a scaled energy estimate for smooth enough solutions of (1.1). We remark that estimate of this type was first proposed by Chemin and Plamchon in [7] for the classical 3-D Navier-Stokes system (see also [6]). The main result of this section states as follows:

Proposition 3.1. Let $s \in\left(\frac{1}{2}, 1\right)$ and $u_{L} \stackrel{\text { def }}{=} e^{t \Delta_{\mathrm{h}}} u_{0}$. Let $u \stackrel{\text { def }}{=} u_{L}+w$ be a smooth enough solution of (1.1) on $\left[0, T^{*}\right)$. Then for any $t<T^{*}$, one has

$$
\begin{aligned}
& \frac{\|w(t)\|_{L^{2}}^{2}}{t^{s}}+\int_{0}^{t}\left(\frac{s}{4} \frac{\left\|w\left(t^{\prime}\right)\right\|_{L^{2}}^{2}}{\left(t^{\prime}\right)^{1+s}}+\frac{\left\|\nabla_{\mathrm{h}} w\left(t^{\prime}\right)\right\|_{L^{2}}^{2}}{\left(t^{\prime}\right)^{s}}\right) d t^{\prime} \\
\leq & C_{s} t^{s-\frac{1}{2}}\left(\left\|u_{0}\right\|_{H^{s}}^{2}+\left\|\partial_{3} u_{0}\right\|_{\dot{H}^{s-1,0}}^{2}\right)\left\|u_{0}\right\|_{H^{s}}^{2} \exp \left(C_{s} t^{\frac{2 s-1}{3}}\left(\left\|u_{0}\right\|_{\dot{H}^{s}}^{\frac{4}{3}}+\left\|\partial_{3} u_{0}\right\|_{\dot{H}^{s-1,0}}^{\frac{4}{3}}\right)\right) .
\end{aligned}
$$

Proof. Due to $u \stackrel{\text { def }}{=} w+u_{L}$, by virtue of (1.1), $w$ verifies

$$
\left\{\begin{array}{l}
\partial_{t} w+\left(u_{L}+w\right) \cdot \nabla w+w \cdot \nabla u_{L}-\Delta_{\mathrm{h}} w=-u_{L} \cdot \nabla u_{L}-\nabla p \\
\operatorname{div} w=0 \\
\left.w\right|_{t=0}=0
\end{array}\right.
$$

By taking $L^{2}$ inner product of (3.2) with $w$ and dividing the resulting equality by $t^{S}$, we find

$$
\begin{aligned}
& \frac{1}{2} \frac{d}{d t}\left(\frac{\|w(t)\|_{L^{2}}^{2}}{t^{s}}\right)+\frac{s}{2} \frac{\|w(t)\|_{L^{2}}^{2}}{t^{1+s}}+\frac{\left\|\nabla_{\mathrm{h}} w(t)\right\|_{L^{2}}^{2}}{t^{s}} \\
= & -\frac{\left(w \cdot \nabla u_{L} \mid w\right)_{L^{2}}}{t^{s}}-\frac{\left(u_{L} \cdot \nabla u_{L} \mid w\right)_{L^{2}}}{t^{s}} .
\end{aligned}
$$

In what follows, we shall separate the estimate of the terms on the right-hand side of (3.3) with vertical derivative and without vertical derivative.

Notice that for any $p>2$, we have

$$
\|f\|_{L_{\mathrm{h}}^{\frac{2 p}{p-1}}} \leq C\|f\|_{L_{\mathrm{h}}^{2}}^{1-\frac{1}{p}}\left\|\nabla_{\mathrm{h}} f\right\|_{L_{\mathrm{h}}^{2}}^{\frac{1}{p}}
$$

from which, we infer

$$
\begin{aligned}
&\left|\left(w^{\mathrm{h}} \cdot \nabla_{\mathrm{h}} u_{L} \mid w\right)_{L^{2}}\right| \leq\left\|\nabla_{\mathrm{h}} u_{L}\right\|_{L_{\mathrm{V}}^{\infty}\left(L_{\mathrm{h}}^{p}\right)}\|w\|_{L_{\mathrm{V}}^{2}\left(L_{\mathrm{h}}^{\frac{2 p}{p-1}}\right)}^{2} \\
& \leq C\left\|\nabla_{\mathrm{h}} u_{L}\right\|_{L_{\mathrm{V}}^{\infty}\left(L_{\mathrm{h}}^{p}\right)}\|w\|_{L^{2}}^{2\left(1-\frac{1}{p}\right)}\left\|\nabla_{\mathrm{h}} w\right\|_{L^{2}}^{\frac{2}{p}} \leq C\left\|\nabla_{\mathrm{h}} u_{L}\right\|_{L_{\mathrm{V}}^{\infty}\left(L_{\mathrm{h}}^{p}\right)}^{\frac{p}{p-1}}\|w\|_{L^{2}}^{2}+\frac{1}{8}\left\|\nabla_{\mathrm{h}} w\right\|_{L^{2}}^{2} .
\end{aligned}
$$


In particular, taking $p=4$ in the above inequality gives rise to

$$
\left|\left(w^{\mathrm{h}} \cdot \nabla_{\mathrm{h}} u_{L} \mid w\right)_{L^{2}}\right| \leq C\left\|\nabla_{\mathrm{h}} u_{L}\right\|_{L_{\mathrm{v}}^{\infty}\left(L_{\mathrm{h}}^{4}\right)}^{\frac{4}{3}}\|w\|_{L^{2}}^{2}+\frac{1}{8}\left\|\nabla_{\mathrm{h}} w\right\|_{L^{2}}^{2} .
$$

While observing that $\operatorname{div} w=0$ and

$$
\|f\|_{L_{\mathrm{h}}^{2}\left(L_{\mathrm{v}}^{\infty}\right)} \leq\|f\|_{L^{2}}^{\frac{1}{2}}\left\|\partial_{3} f\right\|_{L^{2}}^{\frac{1}{2}}
$$

we deduce that

$$
\begin{aligned}
\left|\left(w^{3} \partial_{3} u_{L} \mid w\right)_{L^{2}}\right| & \leq\left\|w^{3}\right\|_{L_{\mathrm{h}}^{2}\left(L_{\mathrm{v}}^{\infty}\right)}\left\|\partial_{3} u_{L}\right\|_{L_{\mathrm{h}}^{\infty}\left(L_{\mathrm{v}}^{2}\right)}\|w\|_{L^{2}} \\
& \leq\left\|w^{3}\right\|_{L^{2}}^{\frac{1}{2}}\left\|\partial_{3} w^{3}\right\|_{L^{2}}^{\frac{1}{2}}\left\|\partial_{3} u_{L}\right\|_{L_{\mathrm{h}}^{\infty}\left(L_{\mathrm{v}}^{2}\right)}\|w\|_{L^{2}} \\
& \leq C\left\|\partial_{3} u_{L}\right\|_{L_{\mathrm{h}}^{\infty}\left(L_{\mathrm{v}}^{2}\right)}^{\frac{4}{3}}\|w\|_{L^{2}}^{2}+\frac{1}{8}\left\|\nabla_{\mathrm{h}} w\right\|_{L^{2}}^{2}
\end{aligned}
$$

Finally applying Young's inequality gives rise to

$$
\begin{aligned}
\frac{\left|\left(u_{L} \cdot \nabla u_{L} \mid w\right)_{L^{2}}\right|}{t^{s}} & \leq t^{\frac{1-s}{2}}\left\|u_{L} \cdot \nabla u_{L}\right\|_{L^{2}} \frac{\|w\|_{L^{2}}}{t^{\frac{1+s}{2}}} \\
& \leq \frac{t^{1-s}}{s}\left\|u_{L} \cdot \nabla u_{L}\right\|_{L^{2}}^{2}+\frac{s\|w\|_{L^{2}}^{2}}{4 t^{1+s}} .
\end{aligned}
$$

Inserting the estimates (3.5), (3.6) and (3.7) into (3.3), we achieve

$$
\begin{aligned}
& \frac{d}{d t}\left(\frac{\|w(t)\|_{L^{2}}^{2}}{t^{s}}\right)+\frac{s}{4} \frac{\|w(t)\|_{L^{2}}^{2}}{t^{1+s}}+\frac{\left\|\nabla_{\mathrm{h}} w(t)\right\|_{L^{2}}^{2}}{t^{s}} \\
\leq & C\left(\left\|\nabla_{\mathrm{h}} u_{L}\right\|_{L_{\mathrm{h}}^{4}\left(L_{\mathrm{v}}^{\infty}\right)}^{\frac{4}{3}}+\left\|\partial_{3} u_{L}\right\|_{L_{\mathrm{h}}^{\infty}\left(L_{\mathrm{V}}^{2}\right)}^{\frac{4}{3}} \frac{\|w\|_{L^{2}}^{2}}{t^{s}}+\frac{2}{s} t^{1-s}\left\|u_{L} \cdot \nabla u_{L}\right\|_{L^{2}}^{2} .\right.
\end{aligned}
$$

Applying Gronwall's inequality gives rise to

$$
\begin{aligned}
& \frac{\|w(t)\|_{L^{2}}^{2}}{t^{s}}+\int_{0}^{t}\left(\frac{s}{4} \frac{\left\|w\left(t^{\prime}\right)\right\|_{L^{2}}^{2}}{\left(t^{\prime}\right)^{1+s}}+\frac{\left\|\nabla_{\mathrm{h}} w\left(t^{\prime}\right)\right\|_{L^{2}}^{2}}{\left(t^{\prime}\right)^{S}}\right) d t^{\prime} \\
\leq & \frac{2}{s} \int_{0}^{t}\left(t^{\prime}\right)^{1-s}\left\|u_{L} \cdot \nabla u_{L}\left(t^{\prime}\right)\right\|_{L^{2}}^{2} d t^{\prime} \\
& \times \exp \left(C \int_{0}^{t}\left(\left\|\nabla_{\mathrm{h}} u_{L}\left(t^{\prime}\right)\right\|_{L_{\mathrm{h}}^{4}\left(L_{\mathrm{v}}^{\infty}\right)}^{\frac{4}{3}}+\left\|\partial_{3} u_{L}\left(t^{\prime}\right)\right\|_{L_{\mathrm{h}}^{\infty}\left(L_{\mathrm{V}}^{2}\right)}^{\frac{4}{3}}\right) d t^{\prime}\right) .
\end{aligned}
$$

Let us now handle term by term in (3.8). 
Estimate of $\int_{0}^{t}\left\|\nabla_{\mathrm{h}} u_{L}\left(t^{\prime}\right)\right\|_{L_{\mathrm{h}}^{4}\left(L_{\mathrm{V}}^{\infty}\right)}^{\frac{4}{3}} d t^{\prime}$

We first observe from Lemma 2.4 of [1] and Lemma 2.1 that

$$
\int_{0}^{t}\left\|\nabla_{\mathrm{h}} u_{L}\left(t^{\prime}\right)\right\|_{L_{\mathrm{h}}^{4}\left(L_{\mathrm{v}}^{\infty}\right)}^{\frac{4}{3}} d t^{\prime} \leq C \int_{0}^{t}\left(\sum_{k \in \mathbb{Z}} e^{-c t^{\prime} 2^{2 k}} 2^{\frac{3 k}{2}} \sum_{\ell \in \mathbb{Z}} 2^{\frac{\ell}{2}}\left\|\Delta_{k}^{\mathrm{h}} \Delta_{\ell}^{\mathrm{v}} u_{0}\right\|_{L^{2}}\right)^{\frac{4}{3}} d t^{\prime} .
$$

Yet it follows from Lemma 4.3 of [9] that $\dot{H}^{s}\left(\mathbb{R}^{3}\right) \hookrightarrow \dot{H}_{\mathrm{h}}^{s-\frac{1}{2}}\left(B_{2,1}^{\frac{1}{2}}\right)_{\mathrm{v}}$, so that

$$
\sum_{\ell \in \mathbb{Z}} 2^{\frac{\ell}{2}}\left\|\Delta_{k}^{\mathrm{h}} \Delta_{\ell}^{\mathrm{v}} u_{0}\right\|_{L^{2}} \lesssim c_{k} 2^{-k\left(s-\frac{1}{2}\right)}\left\|u_{0}\right\|_{\dot{H}^{s}}
$$

where $\left(c_{k}\right)_{k \in \mathbb{Z}}$ is a generic element of $\ell^{2}(\mathbb{Z})$ so that $\sum_{k \in \mathbb{Z}} c_{k}^{2}=1$. As a result, we obtain

$$
\int_{0}^{t}\left\|\nabla_{\mathrm{h}} u_{L}\left(t^{\prime}\right)\right\|_{L_{\mathrm{h}}^{4}\left(L_{\mathrm{v}}^{\infty}\right)}^{\frac{4}{3}} d t^{\prime} \leq C \int_{0}^{t}\left(\sum_{k \in \mathbb{Z}} e^{-c t^{\prime} 2^{2 k}} 2^{k(2-s)}\right)^{\frac{4}{3}} d t^{\prime}\left\|u_{0}\right\|_{\dot{H}^{s}}^{\frac{4}{3}} .
$$

On the other hand, it follows from Lemma 2.35 of [1] that

$$
\sup _{t>0}\left(\sum_{k \in \mathbb{Z}} t^{1-\frac{s}{2}} 2^{k(2-s)} e^{-c t 2^{2 k}}\right) \stackrel{\text { def }}{=} M_{s}<\infty .
$$

Hence we obtain

$$
\begin{aligned}
\int_{0}^{t}\left\|\nabla_{\mathrm{h}} u_{L}\left(t^{\prime}\right)\right\|_{L_{\mathrm{h}}^{4}\left(L_{\mathrm{v}}^{\infty}\right)}^{\frac{4}{3}} d t^{\prime} & \leq C M_{S}^{\frac{4}{3}} \int_{0}^{t}\left(t^{\prime}\right)^{-\frac{4}{3}+\frac{2 s}{3}} d t^{\prime}\left\|u_{0}\right\|_{\dot{H}^{s}}^{\frac{4}{3}} \\
& \leq C_{s} t^{\frac{2 s-1}{3}}\left\|u_{0}\right\|_{H^{s}}^{\frac{4}{3}} .
\end{aligned}
$$

Estimate of $\int_{0}^{t}\left\|\partial_{3} u_{L}\left(t^{\prime}\right)\right\|_{L_{\mathrm{h}}^{\infty}\left(L_{\mathrm{v}}^{2}\right)}^{\frac{4}{3}} d t^{\prime}$

Notice that $\dot{H}^{s-1,0} \hookrightarrow L_{\mathrm{v}}^{2}\left(B_{\infty, 2}^{s-2}\right)_{\mathrm{h}^{\prime}}$ we deduce from Theorem 2.34 of [1] that

$$
\begin{aligned}
& \int_{0}^{t}\left\|\partial_{3} u_{L}\left(t^{\prime}\right)\right\|_{L_{\mathrm{h}}^{\infty}\left(L_{\mathrm{v}}^{2}\right)}^{\frac{4}{3}} d t^{\prime} \\
\leq & \left(\int_{0}^{t}\left(t^{\prime}\right)^{2(s-1)} d t^{\prime}\right)^{\frac{1}{3}}\left(\int_{\mathbb{R}_{z}} \int_{0}^{t}\left(t^{\prime}\right)^{1-s}\left\|\partial_{3} u_{L}\left(t^{\prime}, \cdot, z\right)\right\|_{L_{\mathrm{h}}^{\infty}}^{2} d t^{\prime} d z\right)^{\frac{2}{3}} \\
\leq & C_{S} t^{\frac{2 s-1}{3}}\left(\int_{\mathbb{R}_{z}}\left\|\partial_{3} u_{0}\right\|_{\left(B_{\infty, 2}^{s-2}\right)_{\mathrm{h}}}^{2} d z\right)^{\frac{2}{3}} \\
\leq & C_{s} t^{\frac{2 s-1}{3}}\left\|\partial_{3} u_{0}\right\|_{\dot{H}^{s-1,0}}^{\frac{4}{3}}
\end{aligned}
$$


Estimate of $\int_{0}^{t}\left(t^{\prime}\right)^{1-s}\left\|u_{L} \cdot \nabla u_{L}\left(t^{\prime}\right)\right\|_{L^{2}}^{2} d t^{\prime}$

We first get, by applying Hölder's inequality, that

$$
\begin{aligned}
& \int_{0}^{t}\left(t^{\prime}\right)^{1-s}\left\|u_{L}^{\mathrm{h}} \cdot \nabla_{\mathrm{h}} u_{L}\left(t^{\prime}\right)\right\|_{L^{2}}^{2} d t^{\prime} \\
\leq & \left(\int_{0}^{t}\left\|u_{L}^{\mathrm{h}}(t)\right\|_{L_{\mathrm{V}}^{\infty}\left(L_{\mathrm{h}}^{4}\right)}^{4} d t^{\prime}\right)^{\frac{1}{2}}\left(\int_{0}^{t}\left(t^{\prime}\right)^{2(1-s)}\left\|\nabla_{\mathrm{h}} u_{L}\left(t^{\prime}\right)\right\|_{L_{\mathrm{V}}^{2}\left(L_{\mathrm{h}}^{4}\right)}^{4} d t^{\prime}\right)^{\frac{1}{2}} .
\end{aligned}
$$

Observing that $\dot{H}^{s-1,0} \hookrightarrow L_{\mathrm{v}}^{2}\left(B_{4,2}^{s-\frac{3}{2}}\right)_{\mathrm{h}} \hookrightarrow L_{\mathrm{v}}^{2}\left(B_{4,4}^{s-\frac{3}{2}}\right)_{\mathrm{h}^{\prime}}$ and thanks to Theorem 2.34 of [1], we infer

$$
\begin{aligned}
& \left(\int_{0}^{t}\left(t^{\prime}\right)^{2(1-s)}\left\|\nabla_{\mathrm{h}} u_{L}^{\mathrm{h}}\left(t^{\prime}\right)\right\|_{L_{\mathrm{V}}^{2}\left(L_{\mathrm{h}}^{4}\right)}^{4} d t^{\prime}\right)^{\frac{1}{4}} \\
\leq & \left\|\left(\int_{0}^{t}\left(t^{\prime}\right)^{2(1-s)}\left\|\nabla_{\mathrm{h}} u_{L}^{\mathrm{h}}\left(t^{\prime}, \cdot, z\right)\right\|_{L_{\mathrm{h}}^{4}}^{4} d t^{\prime}\right)^{\frac{1}{4}}\right\|_{L_{\mathrm{v}}^{2}} \\
\lesssim & \|\| \nabla_{\mathrm{h}} u_{0}(\cdot, z)\left\|_{\left(B_{4,2}^{s-\frac{3}{2}}\right)_{\mathrm{h}}}\right\|_{L_{\mathrm{V}}^{2}} \lesssim\left\|\nabla_{h} u_{0}\right\|_{H^{s-1,0}} .
\end{aligned}
$$

Whereas we deduce from (3.9) that

$$
\begin{aligned}
\int_{0}^{t}\left\|u_{L}^{\mathrm{h}}(t)\right\|_{L_{\mathrm{h}}^{4}\left(L_{\mathrm{v}}^{\infty}\right)}^{4} d t^{\prime} & \lesssim \int_{0}^{t}\left(\sum_{k \in \mathbb{Z}} e^{-c t 2^{2 k}} 2^{\frac{k}{2}} \sum_{\ell \in \mathbb{Z}} 2^{\frac{\ell}{2}}\left\|\Delta_{k}^{\mathrm{h}} \Delta_{\ell}^{\mathrm{v}} u_{0}\right\|_{L^{2}}\right)^{4} d t \\
& \lesssim \int_{0}^{t}\left(t^{\prime}\right)^{2(s-1)} \sup _{t>0}\left(\sum_{k \in \mathbb{Z}}\left(t^{\prime}\right)^{\frac{1-s}{2}} 2^{k(1-s)}\right)^{4} d t^{\prime}\left\|u_{0}\right\|_{H^{s}}^{4} \\
& \leq C_{s} t^{2 s-1}\left\|u_{0}\right\|_{H^{s}}^{4} .
\end{aligned}
$$

As a result, it comes out

$$
\int_{0}^{t}\left(t^{\prime}\right)^{1-s}\left\|u_{L}^{\mathrm{h}} \cdot \nabla_{\mathrm{h}} u_{L}\left(t^{\prime}\right)\right\|_{L^{2}}^{2} d t^{\prime} \lesssim t^{s-\frac{1}{2}}\left\|u_{0}\right\|_{H^{s}}^{4}
$$

Exactly along the same line, we have

$$
\int_{0}^{t}\left(t^{\prime}\right)^{1-s}\left\|u_{L}^{3} \partial_{3} u_{L}\left(t^{\prime}\right)\right\|_{L^{2}}^{2} d t^{\prime} \lesssim t^{s-\frac{1}{2}}\left\|\partial_{3} u_{0}\right\|_{H^{s-1,0}}^{2}\left\|u_{0}\right\|_{H^{s}}^{2}
$$

By inserting the estimates (3.10), (3.11), (3.13) and (3.14) into (3.8), we achieve (3.1). This finishes the proof of Proposition 3.1. 


\section{The proof of Theorem 1.1}

The goal of this section is to present the proof of Theorem 1.1. The key ingredient will be the following proposition:

Proposition 4.1. Let $s \in(1 / 2,1)$ and $w$ be a smooth enough solution of (3.2) on $\left[0, T^{*}\right)$. Then for any $t<T^{*}$, we have

$$
\begin{aligned}
& \quad \frac{d}{d t}\|w(t)\|_{\dot{H}^{0, s}}^{2}+\left\|\nabla_{\mathrm{h}} w\right\|_{\dot{H}^{0, s}}^{2} \\
& \leq\left\|\nabla u_{L}\right\|_{\dot{H}^{0, s}}^{2}+C\left(\left\|\partial_{3} u_{L}\right\|_{L_{\mathrm{h}}^{\infty}\left(L_{\mathrm{v}}^{2}\right)}^{\frac{4}{3}}\left\|u_{L}\right\|_{\dot{H}^{0, s}}^{\frac{2}{3}}\|w\|_{\dot{H}^{0, s}}^{\frac{4}{3}}\right. \\
& \quad+\left(\left\|u_{L}\right\|_{L_{\mathrm{v}}^{\infty}\left(L^{4}\right)}^{4}+\left\|\nabla_{\mathrm{h}} u_{L}\right\|_{L_{\mathrm{v}}^{\infty}\left(L^{2}\right)}^{2}+\left\|\partial_{3} u_{L}\right\|_{L_{\mathrm{h}}^{\infty}\left(L_{\mathrm{v}}^{2}\right)}^{\frac{4}{3}}\right)\left(\|w\|_{\dot{H}^{0, s}}^{2}+\left\|u_{L}\right\|_{\dot{H}^{0, s}}^{2}\right) \\
& \left.\quad+\left\|\nabla_{\mathrm{h}} w\right\|_{L^{2}}^{2}\left[\|w\|_{\dot{H}^{0, s}}^{\frac{4 s}{2 s-1}}+\left(\left\|u_{L}\right\|_{\dot{H}^{0, s}}\|w\|_{\dot{H}^{0, s}}\right)^{\frac{2 s}{2 s-1}}+\|w\|_{L^{2}}^{2}\|w\|_{\dot{H}^{0, s}}^{\frac{2(2 s+1)}{2 s-1}}\right]\right) .
\end{aligned}
$$

Proof. By applying the operator $\Delta_{\ell}^{\mathrm{v}}$ to (3.2) and then taking $L^{2}$ inner product of the resulting equations with $\Delta_{\ell}^{\mathrm{v}} w$, we find

$$
\begin{aligned}
& \frac{1}{2} \frac{d}{d t}\left\|\Delta_{\ell}^{\mathrm{v}} w(t)\right\|_{L^{2}}^{2}+\left\|\Delta_{\ell}^{\mathrm{v}} \nabla_{h} w\right\|_{L^{2}}^{2} \\
= & -\left(\Delta_{\ell}^{\mathrm{v}}\left(\left(u_{L}+w\right) \cdot \nabla w\right) \mid \Delta_{\ell}^{\mathrm{v}} w\right)_{L^{2}} \\
& -\left(\Delta_{\ell}^{\mathrm{v}}\left(w \cdot \nabla u_{L}\right) \mid \Delta_{\ell}^{\mathrm{v}} w\right)_{L^{2}}-\left(\Delta_{\ell}^{\mathrm{v}}\left(u_{L} \cdot \nabla u_{L}\right) \mid \Delta_{\ell}^{\mathrm{v}} w\right)_{L^{2}} \cdot
\end{aligned}
$$

Let us now handle term by term in (4.2). We first observe that

$$
\left(\Delta_{\ell}^{\mathrm{v}}(w \cdot \nabla w) \mid \Delta_{\ell}^{\mathrm{v}} w\right)_{L^{2}}=\left(\Delta_{\ell}^{\mathrm{v}}\left(w^{\mathrm{h}} \cdot \nabla_{\mathrm{h}} w\right) \mid \Delta_{\ell}^{\mathrm{v}} w\right)_{L^{2}}+\left(\Delta_{\ell}^{\mathrm{v}}\left(w^{3} \partial_{3} w\right) \mid \Delta_{\ell}^{\mathrm{v}} w\right)_{L^{2}}
$$

Applying Lemma 2.2 yields

$$
\begin{aligned}
\left|\left(\Delta_{\ell}^{\mathrm{v}}\left(w^{\mathrm{h}} \cdot \nabla_{\mathrm{h}} w\right) \mid \Delta_{\ell}^{\mathrm{v}} w\right)_{L^{2}}\right| \leq & \left\|\Delta_{\ell}^{\mathrm{v}}\left(w^{\mathrm{h}} \cdot \nabla_{\mathrm{h}} w\right)\right\|_{L_{\mathrm{V}}^{2}\left(L_{\mathrm{h}}^{3}\right)}\left\|\Delta_{\ell}^{\mathrm{v}} w\right\|_{L_{\mathrm{v}}^{2}\left(L_{\mathrm{h}}^{4}\right)} \\
& \lesssim\left\|\Delta_{\ell}^{\mathrm{v}}\left(w^{\mathrm{h}} \cdot \nabla_{\mathrm{h}} w\right)\right\|_{L_{\mathrm{v}}^{2}\left(L_{\mathrm{h}}^{3}\right)}\left\|\Delta_{\ell}^{\mathrm{v}} w\right\|_{L^{2}}^{\frac{1}{2}}\left\|\Delta_{\ell}^{\mathrm{v}} \nabla_{\mathrm{h}} w\right\|_{L^{2}}^{\frac{1}{2}} \\
& \lesssim c_{\ell}^{2}(t) 2^{-2 \ell s}\left(\|w\|_{\dot{H}^{0, s}}\left\|\nabla_{\mathrm{h}} w\right\|_{\dot{H}^{0, s}}^{1+\frac{1}{2 s}}\left\|\nabla_{\mathrm{h}} w\right\|_{L^{2}}^{1-\frac{1}{2 s}}\right. \\
& \left.+\|w\|_{\dot{H}^{0, s}}^{\frac{1}{2}+\frac{1}{4 s}}\left\|\nabla_{\mathrm{h}} w\right\|_{\dot{H}^{0, s}}^{\frac{3}{4}+\frac{1}{4 s}}\left(\|w\|_{L^{2}}\left\|\nabla_{\mathrm{h}} w\right\|_{L^{2}}\right)^{\frac{1}{2}-\frac{1}{4 s}}\right) .
\end{aligned}
$$


Applying Lemma 2.3 to $\left(\Delta_{\ell}^{\mathrm{v}}\left(w^{3} \partial_{3} w\right) \mid \Delta_{\ell}^{\mathrm{v}} w\right)_{L^{2}}$ gives rise to the same estimate. Therefore, we achieve

$$
\begin{aligned}
\left|\left(\Delta_{\ell}^{\mathrm{v}}(w \cdot \nabla w) \mid \Delta_{\ell}^{\mathrm{v}} w\right)_{L^{2}}\right| \lesssim & c_{\ell}^{2}(t) 2^{-2 \ell s}\left(\|w\|_{\dot{H}^{0, s}}\left\|\nabla_{\mathrm{h}} w\right\|_{\dot{H}^{0, s}}^{1+\frac{1}{2 s}}\left\|\nabla_{\mathrm{h}} w\right\|_{L^{2}}^{1-\frac{1}{2 s}}\right. \\
& \left.+\|w\|_{\dot{H}^{0, s}}^{\frac{1}{2}+\frac{1}{4 s}}\left\|\nabla_{\mathrm{h}} w\right\|_{\dot{H}^{0, s}}^{\frac{3}{4 s}+\frac{1}{s}}\left(\|w\|_{L^{2}}\left\|\nabla_{\mathrm{h}} w\right\|_{L^{2}}\right)^{\frac{1}{2}-\frac{1}{4 s}}\right)
\end{aligned}
$$

which implies that

$$
\begin{aligned}
\sum_{\ell \in \mathbb{Z}} 2^{2 \ell s}\left|\left(\Delta_{\ell}^{\mathrm{v}}(w \cdot \nabla w) \mid \Delta_{\ell}^{\mathrm{v}} w\right)_{L^{2}}\right| \lesssim & \|w\|_{\dot{H}^{0, s}}\left\|\nabla_{\mathrm{h}} w\right\|_{L^{2}}^{1-\frac{1}{2 s}}\left\|\nabla_{\mathrm{h}} w\right\|_{\dot{H}^{0, s}}^{1+\frac{1}{2 s}} \\
& +\|w\|_{\dot{H}^{0, s}}^{\frac{1}{2}+\frac{1}{4 s}}\left(\|w\|_{L^{2}}\left\|\nabla_{\mathrm{h}} w\right\|_{L^{2}}\right)^{\frac{1}{2}-\frac{1}{4 s}}\left\|\nabla_{\mathrm{h}} w\right\|_{\dot{H}^{0, s}}^{\frac{3}{4 s}+\frac{1}{4 s}} .
\end{aligned}
$$

Applying Young's inequality gives rise to

$$
\begin{aligned}
& \sum_{\ell \in \mathbb{Z}} 2^{2 \ell s}\left|\left(\Delta_{\ell}^{\mathrm{v}}(w \cdot \nabla w) \mid \Delta_{\ell}^{\mathrm{v}} w\right)_{L^{2}}\right| \\
\leq & C\left(\|w\|_{\dot{H}^{0, s}}^{\frac{4 s}{2 s-1}}+\|w\|_{L^{2}}^{2}\|w\|_{\dot{H}^{0, s}}^{\frac{2(1+2 s)}{2 s-1}}\right)\left\|\nabla_{\mathrm{h}} w\right\|_{L^{2}}^{2}+\frac{1}{8}\left\|\nabla_{\mathrm{h}} w\right\|_{\dot{H}^{0, s}}^{2} .
\end{aligned}
$$

Similarly, we write

$$
\left(\Delta_{\ell}^{\mathrm{v}}\left(u_{L} \cdot \nabla w\right) \mid \Delta_{\ell}^{\mathrm{v}} w\right)_{L^{2}}=\left(\Delta_{\ell}^{\mathrm{v}}\left(u_{L}^{\mathrm{h}} \cdot \nabla_{\mathrm{h}} w\right) \mid \Delta_{\ell}^{\mathrm{v}} w\right)_{L^{2}}+\left(\Delta_{\ell}^{\mathrm{v}}\left(u_{L}^{3} \partial_{3} w\right) \mid \Delta_{\ell}^{\mathrm{v}} w\right)_{L^{2}} .
$$

By applying the law of product (2.8), we get

$$
\begin{aligned}
\left|\left(\Delta_{\ell}^{\mathrm{v}}\left(u_{L}^{h} \cdot \nabla_{\mathrm{h}} w\right) \mid \Delta_{\ell}^{\mathrm{v}} w\right)_{L^{2}}\right| \leq & \left\|\Delta_{\ell}^{\mathrm{v}}\left(u_{L}^{\mathrm{h}} \cdot \nabla_{\mathrm{h}} w\right)\right\|_{L_{\mathrm{v}}^{2}\left(L_{\mathrm{h}}^{\frac{4}{3}}\right)}\left\|\Delta_{\ell}^{\mathrm{v}} w\right\|_{L_{\mathrm{v}}^{2}\left(L_{\mathrm{h}}^{4}\right)} \\
& \lesssim c_{\ell}^{2}(t) 2^{-2 \ell s}\left(\left\|u_{L}\right\|_{L_{\mathrm{v}}^{\infty}\left(L_{\mathrm{h}}^{4}\right)}\|w\|_{\dot{H}^{0, s}}^{\frac{1}{2}}\left\|\nabla_{\mathrm{h}} w\right\|_{\dot{H}^{0, s}}^{\frac{3}{2}}\right. \\
& \left.+\left\|u_{L}\right\|_{\dot{H}^{0, s}}^{\frac{1}{2}}\left\|\nabla_{h} u_{L}\right\|_{\dot{H}^{0, s}}^{\frac{1}{2}}\left\|\nabla_{\mathrm{h}} w\right\|_{L^{2}}^{1-\frac{1}{2 s}}\|w\|_{\dot{H}^{0, s}}^{\frac{1}{2}}\left\|\nabla_{\mathrm{h}} w\right\|_{\dot{H}^{0, s}}^{\frac{1}{2}+\frac{1}{2 s}}\right) .
\end{aligned}
$$

Whereas applying the law of product (2.13) gives

$$
\begin{aligned}
& \left|\left(\Delta_{\ell}^{\mathrm{v}}\left(u_{L}^{3} \partial_{3} w\right) \mid \Delta_{\ell}^{\mathrm{v}} w\right)_{L^{2}}\right| \\
\lesssim & c_{\ell}^{2}(t) 2^{-2 \ell s}\left(\left\|\nabla_{\mathrm{h}} u_{L}\right\|_{L_{\mathrm{v}}^{\infty}\left(L_{\mathrm{h}}^{2}\right)}\|w\|_{\dot{H}^{0, s}}\left\|\nabla_{\mathrm{h}} w\right\|_{\dot{H}^{0, s}}\right. \\
& \left.+\left\|\nabla_{h} u_{L}\right\|_{\dot{H}^{0, s}}\left(\|w\|_{L^{2}}\left\|\nabla_{\mathrm{h}} w\right\|_{L^{2}}\right)^{\frac{1}{2}-\frac{1}{4 s}}\left(\|w\|_{\dot{H}^{0, s}}\left\|\nabla_{\mathrm{h}} w\right\|_{\dot{H}^{0, s}}\right)^{\frac{1}{2}+\frac{1}{4 s}}\right) .
\end{aligned}
$$


As a result, it comes out

$$
\begin{aligned}
& \quad\left|\left(\Delta_{\ell}^{\mathrm{v}}\left(u_{L} \cdot \nabla w\right) \mid \Delta_{\ell}^{\mathrm{v}} w\right)_{L^{2}}\right| \\
& \lesssim c_{\ell}^{2}(t) 2^{-2 \ell s}\left(\left\|u_{L}\right\|_{L_{\mathrm{v}}^{\infty}\left(L_{\mathrm{h}}^{4}\right)}\|w\|_{\dot{H}^{0, s}}^{\frac{1}{2}}\left\|\nabla_{\mathrm{h}} w\right\|_{\dot{H}^{0, s}}^{\frac{3}{2}}+\left\|\nabla_{\mathrm{h}} u_{L}\right\|_{L_{\mathrm{v}}^{\infty}\left(L_{\mathrm{h}}^{2}\right.}\|w\|_{\dot{H}^{0, s}}\left\|\nabla_{\mathrm{h}} w\right\|_{\dot{H}^{0, s}}\right. \\
& \quad+\left\|u_{L}\right\|_{\dot{H}^{0, s}}^{\frac{1}{2}}\left\|\nabla_{h} u_{L}\right\|_{\dot{H}^{0, s}}^{\frac{1}{2}}\left\|\nabla_{\mathrm{h}} w\right\|_{L^{2}}^{1-\frac{1}{2 s}}\|w\|_{\dot{H}^{0, s}}^{\frac{1}{2}}\left\|\nabla_{\mathrm{h}} w\right\|_{\dot{H}^{0, s}}^{\frac{1}{2 s}+\frac{1}{2 s}} \\
& \left.\quad+\left\|\nabla_{h} u_{L}\right\|_{\dot{H}^{0, s}}\left(\|w\|_{L^{2}}\left\|\nabla_{\mathrm{h}} w\right\|_{L^{2}}\right)^{\frac{1}{2}-\frac{1}{4 s}}\left(\|w\|_{\dot{H}^{0, s}}\left\|\nabla_{\mathrm{h}} w\right\|_{\dot{H}^{0, s}}\right)^{\frac{1}{2}+\frac{1}{4 s}}\right) .
\end{aligned}
$$

Then we get, by a similar derivation of (4.3), that

$$
\begin{aligned}
& \sum_{\ell \in \mathbb{Z}} 2^{2 \ell s}\left|\left(\Delta_{\ell}^{\mathrm{v}}\left(u_{L} \cdot \nabla w\right) \mid \Delta_{\ell}^{\mathrm{v}} w\right)_{L^{2}}\right| \\
\leq & C\left(\left\|\nabla_{\mathrm{h}} w\right\|_{L^{2}}^{2}\left[\left(\left\|u_{L}\right\|_{\dot{H}^{0, s}}\|w\|_{\dot{H}^{0, s}}\right)^{\frac{2 s}{2 s-1}}+\|w\|_{L^{2}}^{2}\|w\|_{\dot{H}^{0, s}}^{\frac{2(2 s+1)}{2 s-1}}\right]\right. \\
& \left.+\left(\left\|\nabla_{\mathrm{h}} u_{L}\right\|_{L_{\mathrm{v}}^{\infty}\left(L^{2}\right)}^{2}+\left\|u_{L}\right\|_{L_{\mathrm{V}}^{\infty}\left(L^{4}\right)}^{4}\right)\|w\|_{\dot{H}^{0, s}}^{2}\right)+\frac{1}{8}\left(\left\|\nabla_{\mathrm{h}} w\right\|_{\dot{H}^{0, s}}^{2}+\left\|\nabla_{h} u_{L}\right\|_{\dot{H}^{0, s}}^{2}\right) .
\end{aligned}
$$

To handle the last terms in (4.2) involving horizontal derivatives, we apply Remark 2.1 to get

$$
\begin{aligned}
& \left|\left(\Delta_{\ell}^{\mathrm{v}}\left(w \cdot \nabla_{\mathrm{h}} u_{L}\right) \mid \Delta_{\ell}^{\mathrm{v}} w\right)_{L^{2}}\right| \\
\leq & \left\|\Delta_{\ell}^{\mathrm{v}}\left(w \cdot \nabla_{\mathrm{h}} u_{L}\right)\right\|_{L_{\mathrm{v}}^{2}\left(L_{\mathrm{h}}^{\frac{4}{3}}\right)}\left\|\Delta_{\ell}^{\mathrm{v}} w\right\|_{L_{\mathrm{V}}^{2}\left(L_{\mathrm{h}}^{4}\right)} \\
\lesssim & c_{\ell}^{2}(t) 2^{-2 \ell s}\left(\left\|\nabla_{\mathrm{h}} u_{L}\right\|_{L_{\mathrm{V}}^{\infty}\left(L^{2}\right)}\|w\|_{\dot{H}^{0, s}}\left\|\nabla_{\mathrm{h}} w\right\|_{\dot{H}^{0, s}}\right. \\
& \left.+\left(\|w\|_{L^{2}}\left\|\nabla_{\mathrm{h}} w\right\|_{L^{2}}\right)^{\frac{1}{2}-\frac{1}{4 s}}\left(\|w\|_{\dot{H}^{0, s}}\left\|\nabla_{h} w\right\|_{\dot{H}^{0, s}}\right)^{\frac{1}{2}+\frac{1}{4 s}}\left\|\nabla_{\mathrm{h}} u_{L}\right\|_{\dot{H}^{0, s}}\right),
\end{aligned}
$$

and

$$
\begin{aligned}
\left|\left(\Delta_{\ell}^{\mathrm{v}}\left(u_{L} \cdot \nabla_{\mathrm{h}} u_{L}\right) \mid \Delta_{\ell}^{\mathrm{v}} w\right)_{L^{2}}\right| \leq & \left\|\Delta_{\ell}^{\mathrm{v}}\left(u_{L} \cdot \nabla_{\mathrm{h}} u_{L}\right)\right\|_{L_{\mathrm{v}}^{2}\left(L_{\mathrm{h}}^{3}\right)}\left\|\Delta_{\ell}^{\mathrm{v}} w\right\|_{L_{\mathrm{v}}^{2}\left(L_{\mathrm{h}}^{4}\right)} \\
\leq & c_{\ell}^{2}(t) 2^{-2 \ell s}\left(\left\|\nabla_{\mathrm{h}} u_{L}\right\|_{L_{\mathrm{v}}^{\infty}\left(L^{2}\right)}\left\|u_{L}\right\|_{\dot{H}^{0, s}}^{\frac{1}{2}}\left\|\nabla_{h} u_{L}\right\|_{\dot{H}^{0, s}}^{\frac{1}{2}}\right. \\
& \left.+\left\|u_{L}\right\|_{L_{\mathrm{v}}^{\infty}\left(L_{\mathrm{h}}^{4}\right)}\left\|\nabla_{\mathrm{h}} u_{L}\right\|_{\dot{H}^{0, s}}\right)\|w\|_{\dot{H}^{0, s}}^{\frac{1}{2}}\left\|\nabla_{\mathrm{h}} w\right\|_{\dot{H}^{0, s}}^{\frac{1}{2}}
\end{aligned}
$$

Then a similar derivation of (4.3) gives rise to

$$
\begin{aligned}
& \sum_{\ell \in \mathbb{Z}} 2^{2 \ell s}\left|\left(\Delta_{\ell}^{\mathrm{v}}\left(w \cdot \nabla_{h} u_{L}\right) \mid \Delta_{\ell}^{\mathrm{v}} w\right)_{L^{2}}\right| \\
\leq & C\left(\left\|\nabla_{\mathrm{h}} u_{L}\right\|_{L_{\mathrm{v}}^{\infty}\left(L^{2}\right)}^{2}\|w\|_{\dot{H}^{0, s}}^{2}+\|w\|_{L^{2}}^{2}\left\|\nabla_{\mathrm{h}} w\right\|_{L^{2}}^{2}\|w\|_{\dot{H}^{0, s}}^{\frac{2(2 s+1)}{2 s-1}}\right) \\
& +\frac{1}{8}\left(\left\|\nabla_{\mathrm{h}} w\right\|_{\dot{H}^{0, s}}^{2}+\left\|\nabla_{\mathrm{h}} u_{L}\right\|_{\dot{H}^{0, s}}^{2}\right),
\end{aligned}
$$


and

$$
\begin{aligned}
& \sum_{\ell \in \mathbb{Z}} 2^{2 \ell s}\left|\left(\Delta_{\ell}^{\mathrm{v}}\left(u_{L} \cdot \nabla_{\mathrm{h}} u_{L}\right) \mid \Delta_{\ell}^{\mathrm{v}} w\right)_{L^{2}}\right| \\
\leq & C\left(\left(\left\|u_{L}\right\|_{L_{\mathrm{v}}^{\infty}\left(L^{4}\right)}^{4}+\left\|\nabla_{\mathrm{h}} u_{L}\right\|_{L_{\mathrm{v}}^{\infty}\left(L^{2}\right)}^{2}\right)\|w\|_{\dot{H}^{0, s}}^{2}+\left\|\nabla u_{L}\right\|_{L_{\mathrm{v}}^{\infty}\left(L^{2}\right)}^{2}\left\|u_{L}\right\|_{\dot{H}^{0, s}}^{2}\right) \\
& +\frac{1}{8}\left(\left\|\nabla_{\mathrm{h}} w\right\|_{\dot{H}^{0, s}}^{2}+\left\|\nabla_{\mathrm{h}} u_{L}\right\|_{\dot{H}^{0, s}}^{2}\right) .
\end{aligned}
$$

To handle the last two terms in (4.2) involving vertical derivative, we need the following lemma:

Lemma 4.1. Let $a=\left(a^{\mathrm{h}}, a^{3}\right)$ be a solenoidal vector field. Then we have

$$
\begin{aligned}
\left|\left(\Delta_{\ell}^{\mathrm{v}}\left(a^{3} \partial_{3} u_{L}\right) \mid \Delta_{\ell}^{\mathrm{v}} b\right)_{L^{2}}\right| \lesssim & c_{\ell}^{2} 2^{-2 \ell s}\left(\left\|a^{3}\right\|_{L_{\mathrm{v}}^{\infty}\left(L_{\mathrm{h}}^{4}\right)}\left\|\partial_{3} u_{L}\right\|_{\dot{H}^{0, s}}\|b\|_{\dot{H}^{0, s}}^{\frac{1}{2}}\left\|\nabla_{\mathrm{h}} b\right\|_{\dot{H}^{0, s}}^{\frac{1}{2}}\right. \\
& \left.+\left\|\partial_{3} u_{L}\right\|_{L_{\mathrm{h}}^{\infty}\left(L_{\mathrm{v}}^{2}\right)}\left\|a^{3}\right\|_{\dot{H}^{0, s}}^{\frac{1}{2}}\left\|\nabla_{\mathrm{h}} a^{\mathrm{h}}\right\|_{\dot{H}^{0, s}}^{\frac{1}{2}}\|b\|_{\dot{H}^{0, s}}\right) .
\end{aligned}
$$

Let us postpone the proof of this lemma till we finish the proof of this proposition.

Applying (2.4) and Lemma 4.1 yields

$$
\begin{aligned}
& \left|\left(\Delta_{\ell}^{\mathrm{v}}\left(w^{3} \cdot \partial_{3} u_{L}\right) \mid \Delta_{\ell}^{\mathrm{v}} w\right)_{L^{2}}\right| \\
\lesssim & c_{\ell}^{2}(t) 2^{-2 \ell s}\left(\left\|\partial_{3} u_{L}\right\|_{L_{\mathrm{h}}^{\infty}\left(L_{\mathrm{v}}^{2}\right.}\right)\|w\|_{\dot{H}^{0, s}}^{\frac{3}{2}}\left\|\nabla_{\mathrm{h}} w^{\mathrm{h}}\right\|_{\dot{H}^{0, s}}^{\frac{1}{2}} \\
& \left.+\left(\left\|w^{3}\right\|_{L^{2}}\left\|\nabla_{\mathrm{h}} w^{3}\right\|_{L^{2}}\right)^{\frac{1}{2}-\frac{1}{4 s}}\left\|\partial_{3} u_{L}\right\|_{\dot{H}^{0, s}}\left(\|w\|_{\dot{H}^{0, s}}\left\|\nabla_{\mathrm{h}} w\right\|_{\dot{H}^{0, s}}\right)^{\frac{1}{2}+\frac{1}{4 s}}\right),
\end{aligned}
$$

and

$$
\begin{aligned}
\left|\left(\Delta_{\ell}^{\mathrm{v}}\left(u_{L}^{3} \partial_{3} u_{L}\right) \mid \Delta_{\ell}^{\mathrm{v}} w\right)_{L^{2}}\right| \lesssim & c_{\ell}^{2}(t) 2^{-2 \ell s}\left(\left\|u_{L}^{3}\right\|_{L_{\mathrm{v}}^{\infty}\left(L_{\mathrm{h}}^{4}\right)}\left\|\partial_{3} u_{L}\right\|_{\dot{H}^{0, s}}\|w\|_{\dot{H}^{0, s}}^{\frac{1}{2}}\left\|\nabla_{\mathrm{h}} w\right\|_{\dot{H}^{0, s}}^{\frac{1}{2}}\right. \\
& \left.+\left\|\partial_{3} u_{L}\right\|_{L_{\mathrm{h}}^{\infty}\left(L_{\mathrm{v}}^{2}\right)}\left\|u_{L}^{3}\right\|_{\dot{H}^{0, s}}^{\frac{1}{2}}\left\|\nabla_{\mathrm{h}} u_{L}^{\mathrm{h}}\right\|_{\dot{H}^{0, s}}^{\frac{1}{2}}\|w\|_{\dot{H}^{0, s}}\right) .
\end{aligned}
$$

Then a similar derivation of (4.3) gives rise to

$$
\begin{aligned}
& \sum_{\ell \in \mathbb{Z}} 2^{2 \ell s}\left|\left(\Delta_{\ell}^{\mathrm{v}}\left(w^{3} \partial_{3} u_{L}\right) \mid \Delta_{\ell}^{\mathrm{v}} w\right)_{L^{2}}\right| \\
\leq & C\left(\left\|\partial_{3} u_{L}\right\|_{L_{\mathrm{h}}^{\infty}\left(L_{\mathrm{v}}^{2}\right)}^{\frac{4}{3}}\|w\|_{\dot{H}^{0, s}}^{2}+\left\|w^{3}\right\|_{L^{2}}^{2}\left\|\nabla_{\mathrm{h}} w\right\|_{L^{2}}^{2}\|w\|_{\dot{H}^{0, s}}^{\frac{2(2 s+1)}{2 s-1}}\right) \\
& +\frac{1}{8}\left(\left\|\partial_{3} u_{L}\right\|_{\dot{H}^{0, s}}^{2}+\left\|\nabla_{\mathrm{h}} w\right\|_{\dot{H}^{0, s}}^{2}\right),
\end{aligned}
$$


and

$$
\begin{aligned}
& \sum_{\ell \in \mathbb{Z}} 2^{2 \ell s}\left|\left(\Delta_{\ell}^{\mathrm{v}}\left(u_{L}^{3} \partial_{3} u_{L}\right) \mid \Delta_{\ell}^{\mathrm{v}} w\right)_{L^{2}}\right| \\
\leq & C\left(\left\|u_{L}\right\|_{L_{\mathrm{v}}^{\infty}\left(L^{4}\right)}^{4}\|w\|_{\dot{H}^{0, s}}^{2}+\left\|\partial_{3} u_{L}\right\|_{L_{\mathrm{h}}^{\infty}\left(L_{\mathrm{v}}^{2}\right)}^{\frac{4}{3}}\left\|u_{L}\right\|_{\dot{H}^{0, s}}^{\frac{2}{3}}\|w\|_{\dot{H}^{0, s}}^{3}\right. \\
& +\frac{1}{8}\left(\left\|\nabla_{h} u_{L}\right\|_{\dot{H}^{0, s}}^{2}+\left\|\nabla_{\mathrm{h}} w\right\|_{\dot{H}^{0, s}}^{2}+\left\|\partial_{3} u_{L}\right\|_{\dot{H}^{0, s}}^{2}\right) .
\end{aligned}
$$

Multiplying (4.2) by $2^{2 \ell s}$ and summing up the resulting inequalities over $\ell \in Z$, and then inserting the estimates (4.3), (4.4), (4.5), (4.6), (4.8) and (4.9) into the resulting inequality, we obtain (4.1). This completes the proof.

Proposition 4.1 is proved provided that we present the proof of Lemma 4.1.

Proof of Lemma 4.1. We first get, by applying Bony's decomposition (2.2) in the vertical variable $a^{3} \partial_{3} u_{L}$, that

$$
a^{3} \partial_{3} u_{L}=T_{a^{3}}^{\mathrm{v}} \partial_{3} u_{L}+R^{\mathrm{v}}\left(a^{3}, \partial_{3} u_{L}\right) .
$$

Considering the support properties to the Fourier transform of the terms in $T_{a^{3}}^{v} \partial_{3} u_{L}$, we infer

$$
\begin{aligned}
\left|\left(\Delta_{\ell}^{\mathrm{v}}\left(T_{a^{3}}^{\mathrm{v}} \partial_{3} u_{L}\right) \mid \Delta_{\ell}^{\mathrm{v}} b\right)_{L^{2}}\right| & \lesssim \sum_{|j-\ell| \leq 5}\left\|S_{j-1}^{\mathrm{v}} a^{3}\right\|_{L_{\mathrm{v}}^{\infty}\left(L_{\mathrm{h}}^{4}\right)}\left\|\Delta_{j}^{\mathrm{v}} \partial_{3} u_{L}\right\|_{L^{2}}\left\|\Delta_{\ell}^{\mathrm{v}} b\right\|_{L_{\mathrm{v}}^{2}\left(L_{\mathrm{h}}^{4}\right)} \\
& \lesssim \sum_{|j-\ell| \leq 5}\left\|a^{3}\right\|_{L_{\mathrm{v}}^{\infty}\left(L_{\mathrm{h}}^{4}\right)}\left\|\Delta_{j}^{\mathrm{v}} \partial_{3} u_{L}\right\|_{L^{2}}\left\|\Delta_{\ell}^{\mathrm{v}} b\right\|_{L^{2}}^{\frac{1}{2}}\left\|\Delta_{\ell}^{\mathrm{v}} \nabla_{\mathrm{h}} b\right\|_{L^{2}}^{\frac{1}{2}} \\
& \lesssim c_{\ell}^{2} 2^{-2 \ell s}\left\|a^{3}\right\|_{L_{\mathrm{v}}^{\infty}\left(L_{\mathrm{h}}^{4}\right)}\left\|\partial_{3} u_{L}\right\|_{\dot{H}^{0, s}}\|b\|_{\dot{H}^{0, s}}^{\frac{1}{2}}\left\|\nabla_{\mathrm{h}} b\right\|_{\dot{H}^{0, s}}^{\frac{1}{2}}
\end{aligned}
$$

Along the same line, due to $\partial_{3} a^{3}=-\operatorname{div}_{\mathrm{h}} a^{\mathrm{h}}$, we deduce that

$$
\begin{aligned}
& \left|\left(\Delta_{\ell}^{\mathrm{v}}\left(R^{\mathrm{v}}\left(a^{3}, \partial_{3} u_{L}\right)\right) \mid \Delta_{\ell}^{\mathrm{v}} b\right)_{L^{2}}\right| \\
\lesssim & \sum_{j \geq \ell-N_{0}}\left\|\Delta_{j}^{\mathrm{v}} a^{3}\right\|_{L_{\mathrm{h}}^{2}\left(L_{\mathrm{v}}^{\infty}\right)}\left\|S_{j+2}^{\mathrm{v}} \partial_{3} u_{L}\right\|_{L_{\mathrm{h}}^{\infty}\left(L_{\mathrm{v}}^{2}\right)}\left\|\Delta_{\ell}^{\mathrm{v}} b\right\|_{L^{2}} \\
\lesssim & \sum_{j \geq \ell-N_{0}}\left\|\Delta_{j}^{\mathrm{v}} a^{3}\right\|_{L^{2}}^{\frac{1}{2}}\left\|\Delta_{j}^{\mathrm{v}} \partial_{3} a^{3}\right\|_{L^{2}}^{\frac{1}{2}}\left\|\partial_{3} u_{L}\right\|_{L_{\mathrm{h}}^{\infty}\left(L_{\mathrm{v}}^{2}\right)}\left\|\Delta_{\ell}^{\mathrm{v}} b\right\|_{L^{2}}^{\frac{1}{2}}\left\|\Delta_{\ell}^{\mathrm{v}} \nabla_{\mathrm{h}} b\right\|_{L^{2}}^{\frac{1}{2}} \\
\lesssim & c_{\ell}^{2} 2^{-2 \ell s}\left\|\partial_{3} u_{L}\right\|_{L_{\mathrm{h}}^{\infty}\left(L_{\mathrm{v}}^{2}\right)}\left\|a^{3}\right\|_{\dot{H}^{0, s}}^{\frac{1}{2}}\left\|\nabla_{\mathrm{h}} a^{\mathrm{h}}\right\|_{\dot{H}^{0, s}}^{\frac{1}{2}}\|b\|_{\dot{H}^{0, s}} .
\end{aligned}
$$

This leads to (4.7). 
Now we are in a position to complete the proof of Theorem 1.1.

Proof of Theorem 1.1. The local well-posedness part of Theorem 1.1 has been proved in $[4,11]$. Let us denote $T^{*}\left(u_{0}\right)$ be the maximal existence of such a solution. It remains to prove (1.4). Indeed, we first get, by a similar derivation of (3.10) that

$$
\begin{aligned}
\int_{0}^{t}\left\|\nabla_{\mathrm{h}} u_{L}\left(t^{\prime}\right)\right\|_{L_{\mathrm{h}}^{2}\left(L_{\mathrm{v}}^{\infty}\right)}^{2} d t^{\prime} & \leq C \int_{0}^{t}\left(\sum_{k \in \mathbb{Z}} e^{-c t^{\prime} 2^{2 k}} 2^{k} \sum_{\ell \in \mathbb{Z}} 2^{\frac{\ell}{2}}\left\|\Delta_{k}^{\mathrm{h}} \Delta_{\ell}^{\mathrm{v}} u_{0}\right\|_{L^{2}}\right)^{2} d t^{\prime} \\
& \leq C \int_{0}^{t}\left(t^{\prime}\right)^{s-\frac{3}{2}} d t^{\prime}\left\|u_{0}\right\|_{H^{s}}^{2} \sup _{t^{\prime} \in[0, t]}\left(\sum_{k \in \mathbb{Z}} e^{-c t^{\prime} 2^{2 k}}\left(t^{\prime}\right)^{\frac{3}{4}-\frac{s}{2}} 2^{k\left(\frac{3}{2}-s\right)}\right)^{2} \\
& \leq C_{s} t^{s-\frac{1}{2}}\left\|u_{0}\right\|_{H^{s}}^{2} .
\end{aligned}
$$

Now let us define

$$
\begin{gathered}
T_{1}^{\star} \stackrel{\text { def }}{=} \sup \left\{T \leq T^{*}\left(u_{0}\right) ; \sup _{0 \leq t \leq T}\left(\|w(t)\|_{\dot{H}^{0, s}}^{2}+\left\|\nabla_{\mathrm{h}} w\right\|_{L_{t}^{2}\left(\dot{H}^{0, s}\right)}^{2}\right) \leq 4\left[u_{0}\right]_{s}^{2}\right\} \\
\quad \text { with } \quad\left[u_{0}\right]_{s}^{2} \stackrel{\text { def }}{=}\left\|u_{0}\right\|_{\dot{H}^{0, s}}^{2}+\left\|\partial_{3} u_{0}\right\|_{\dot{H}^{s-1,0}}^{2}+\left\|\partial_{3} u_{0}\right\|_{\dot{H}^{-1, s}}^{2} \cdot
\end{gathered}
$$

Then for $t \leq T_{1}^{\star}$, by virtue of (3.11) and (3.12), we have

$$
\int_{0}^{t}\left\|\partial_{3} u_{L}\right\|_{L_{\mathrm{h}}^{\infty}\left(L_{\mathrm{v}}^{2}\right)}^{\frac{4}{3}}\left\|u_{L}\right\|_{\dot{H}^{0, s}}^{\frac{2}{3}}\|w\|_{\dot{H}^{0, s}}^{\frac{4}{3}} d t^{\prime} \leq C_{s} t^{\frac{2 s-1}{3}}\left\|\partial_{3} u_{0}\right\|_{\dot{H}^{s-1,0}}^{\frac{4}{3}}\left[u_{0}\right]_{s}^{2}
$$

and

$$
\begin{aligned}
& \int_{0}^{t}\left(\left\|u_{L}\right\|_{L_{\mathrm{v}}^{\infty}\left(L^{4}\right)}^{4}+\left\|\nabla_{\mathrm{h}} u_{L}\right\|_{L_{\mathrm{v}}^{\infty}\left(L^{2}\right)}^{2}+\left\|\partial_{3} u_{L}\right\|_{L_{\mathrm{v}}^{\infty}\left(L^{2}\right)}^{\frac{4}{3}}\right)\left(\|w\|_{\dot{H}^{0, s}}^{2}+\left\|u_{L}\right\|_{\dot{H}^{0, s}}^{2}\right) d t^{\prime} \\
\leq & C_{s}\left(t^{2 s-1}\left\|u_{0}\right\|_{H^{s}}^{4}+t^{\frac{2 s-1}{3}}\left\|\partial_{3} u_{0}\right\|_{H^{s-1,0}}^{\frac{4}{3}}+t^{s-\frac{1}{2}}\left\|u_{0}\right\|_{H^{s}}^{2}\right)\left[u_{0}\right]_{s}^{2} .
\end{aligned}
$$

In order to deal with the integral of the last term in (4.1), we define

$$
T_{2}^{\star} \stackrel{\text { def }}{=} \min \left(T_{1}^{\star}, \varepsilon\left[u_{0}\right]_{s}^{-\frac{1}{\gamma}}\right) .
$$

Then for $t \leq T_{2}^{\star}$, we deduce from Proposition 3.1 that

$$
\frac{\|w(t)\|_{L^{2}}^{2}}{t^{s}}+\int_{0}^{t} \frac{\left\|\nabla_{\mathrm{h}} w\left(t^{\prime}\right)\right\|_{L^{2}}^{2}}{\left(t^{\prime}\right)^{s}} d t^{\prime} \leq C t^{s-\frac{1}{2}}\left[u_{0}\right]_{s}^{4} .
$$


As a result, we find

$$
\begin{aligned}
& \int_{0}^{t}\left\|\nabla_{\mathrm{h}} w\right\|_{L^{2}}^{2}\left[\|w\|_{\dot{H}^{0, s}}^{\frac{4 s}{2 s-1}}+\left(\left\|u_{L}\right\|_{\dot{H}^{0, s}}\|w\|_{\dot{H}^{0, s}}\right)^{\frac{2 s}{2 s-1}}+\|w\|_{L^{2}}^{2}\|w\|_{\dot{H}^{0, s}}^{\frac{2(2 s+1)}{2 s-1}}\right] d t^{\prime} \\
\leq & C\left(t^{s}\left[u_{0}\right]_{\dot{H}^{0, s}}^{\frac{4 s}{2 s-1}}+C t^{2 s}\left[u_{0}\right]_{\dot{H}^{0, s}}^{\frac{2(2 s+1)}{2 s-1}} \sup _{t^{\prime} \in[0, t]} \frac{\left\|w\left(t^{\prime}\right)\right\|_{L^{2}}^{2}}{\left(t^{\prime}\right)^{s}}\right) \int_{0}^{t} \frac{\left\|\nabla_{\mathrm{h}} w\left(t^{\prime}\right)\right\|_{L^{2}}^{2}}{\left(t^{\prime}\right)^{s}} d t^{\prime} \\
\leq & C\left(t^{2 s-\frac{1}{2}}\left[u_{0}\right]_{s}^{\frac{4 s}{2 s-1}}+t^{4 s-1}\left[u_{0}\right]_{s}^{\frac{12 s-2}{2 s-1}}\right)\left[u_{0}\right]_{s}^{4} .
\end{aligned}
$$

Let $s=\frac{1}{2}+2 \gamma$ for $\gamma \in(0,1 / 4)$, for $t \leq T_{2}^{\star}$, by integrating (4.1) over $[0, t]$ and then inserting the estimates, (4.12), (4.13) and (4.16), into the resulting inequality, we obtain

$$
\begin{aligned}
& \|w(t)\|_{\dot{H}^{0, s}}^{2}+\left\|\nabla_{\mathrm{h}} w\right\|_{L_{t}^{2}\left(\dot{H}^{0, s}\right)}^{2} \\
\leq & 2\left\|u_{0}\right\|_{\dot{H}^{0, s}}^{2}+\left\|\partial_{3} u_{0}\right\|_{\dot{H}^{-1, s}}^{2} \\
& +C_{s}\left(t^{\frac{4 \gamma}{3}}\left[u_{0}\right]_{s}^{\frac{4}{3}}+t^{2 \gamma}\left[u_{0}\right]_{s}^{2}+t^{\frac{1+8 \gamma}{2}}\left[u_{0}\right]_{s}^{\frac{1+8 \gamma}{2 \gamma}}+t^{1+8 \gamma}\left[u_{0}\right]_{s}^{\frac{1+8 \gamma}{\gamma}}\right)\left[u_{0}\right]_{s}^{2} .
\end{aligned}
$$

Therefore as long as $\varepsilon$ is sufficiently small in (4.14), we deduce from (4.17) that

$$
\|w(t)\|_{\dot{H}^{0, s}}^{2}+\left\|\nabla_{\mathrm{h}} w\right\|_{L_{t}^{2}\left(\dot{H}^{0, s}\right)}^{2} \leq 3\left[u_{0}\right]_{s}^{2} \quad \text { for } \quad t \leq T_{2}^{\star} .
$$

Then a standard continuous argument shows that $T_{1}^{\star} \geq \varepsilon\left[u_{0}\right]_{s}^{-\frac{1}{\gamma}}$. Hence we conclude that

$$
T^{*}\left(u_{0}\right) \geq \varepsilon\left[u_{0}\right]_{s}^{-\frac{1}{\gamma}}
$$

This completes the proof of Theorem 1.1.

\section{Acknowledgments}

S. Liang is grateful to the financial supports of the DFG through IRTG 2235. P. Zhang is partially supported by NSF of China under Grants 11371347 and 11688101, and innovation grant from National Center for Mathematics and Interdisciplinary Sciences. 


\section{References}

[1] H. Bahouri, J.-Y. Chemin and R. Danchin, Fourier Analysis and Nonlinear Partial Differential Equations, Grundlehren der mathematischen Wissenschaften, 343, SpringerVerlag, Berlin-Heidelberg, 2011.

[2] J.-M. Bony, Calcul symbolique et propagation des singularités pour les équations aux dérivées partielles non linéaires, Ann. Sci. Éc. Norm. Supér., 14 (1981), 209-246.

[3] J.-Y. Chemin, Anisotropic phenomena in incompressible Navier-Stokes equations, Lecture notes in the Morningside Center of the The Chinese Academy of Sciences, 2018.

[4] J.-Y. Chemin, B. Desjardins, I. Gallagher and E. Grenier, Fluids with anisotropic viscosity, M2AN Math. Model. Numer. Anal., 34 (2000), 315-335.

[5] J.-Y. Chemin, B. Desjardins, I. Gallagher and E. Grenier, Mathematical Geophysics. An introduction to rotating fluids and the Navier-Stokes equations. Oxford Lecture Series in Mathematics and its Applications, 32, The Clarendon Press, Oxford University Press, Oxford, 2006.

[6] J.-Y. Chemin and I. Gallagher, A non-linear estimate on the life span of solutions of the three dimensional Navier-Stokes equations, Tunis. J. Math., 1 (2019), 273-293.

[7] J.-Y. Chemin and F. Planchon, Self-improving bounds for the Navier-Stokes equations, Bull. Soc. Math. France, 140 (2012), 583-597.

[8] J.-Y. Chemin and P. Zhang, On the global wellposedness to the 3-D incompressible anisotropic Navier-Stokes equations, Comm. Math. Phys., 272 (2007), 529-566.

[9] J.-Y. Chemin and P. Zhang, On the critical one component regularity for 3-D NavierStokes system, Ann. Sci. Éc. Norm. Supér., 49 (2016), 131-167.

[10] H. Fujita and T. Kato, On the Navier-Stokes initial value problem I, Arch. Ration. Mech. Anal., 16 (1964), 269-315.

[11] D. Iftimie, The resolution of the Navier-Stokes equations in anisotropic spaces, Rev. Mat. Iberoamericana, 15 (1999), 1-36.

[12] J. Leray, Essai sur le mouvement d'un liquide visqueux emplissant l'espace, Acta Math., 63 (1933), 193-248.

[13] Y. Liu, M. Paicu and P. Zhang, Global well-posedness of 3-D anisotropic NavierStokes system with small unidirectional derivative, arXiv:1905.00156.

[14] M. Paicu, Équation anisotrope de Navier-Stokes dans des espaces critiques, Rev. Mat. Iberoam., 21 (2005), 179-235.

[15] M. Paicu and P. Zhang, Global solutions to the 3-D incompressible anisotropic Navier-Stokes system in the critical spaces, Comm. Math. Phys., 307 (2011), 713-759.

[16] J. Pedlosky, Geophysical Fluid Dynamics. Berlin-Heidelberg-NewYork: Springer, 1979.

[17] P. Zhang, Global Fujita-Kato solution of 3-D inhomogeneous incompressible Navier-Stokes system, Adv. Math., 363 (2020), 107007. 